\title{
Full electric-field tuning of the nonreciprocal transport effect in massive chiral fermions with trigonal warping
}

\author{
Elisha Cho-Hao Lu $\odot$, Cheng-Tung Cheng, Liang Li $\odot$, and Wei-Li Lee $\odot^{*}$ \\ Institute of Physics, Academia Sinica, Nankang, Taipei 11529, Taiwan, Republic of China
}

(Received 12 April 2021; revised 28 July 2021; accepted 29 July 2021; published 16 August 2021)

\begin{abstract}
In a noncentrosymmetric system, an intrinsic electric polarization is allowed and may lead to unusual nonreciprocal charge transport phenomena. As a result, a current-dependent resistance, arising from the magnetoelectric anisotropy term of $k \cdot \vec{E} \times \vec{B}$, appears and acts as a current rectifier with the amount of rectification being linearly proportional to the magnitude of both current and applied magnetic field. In this work, a different type of nonreciprocal transport effect was demonstrated in a graphene-based device, which requires no external magnetic field. Due to the unique pseudospin (valley) degree of freedom in chiral fermions with trigonal warping, a large nonreciprocal transport effect was uncovered in a gapped bilayer graphene, where electric-field tunabilities of the band gap and valley polarization play an important role. The exact cancellation of nonreciprocal effect between two different valleys is effectively removed by breaking the inversion symmetry via electric gatings. The magnitude of the current rectification appears to be at a maximum when the Fermi surface undergoes a Lifshitz transition near the band edges, which is proportional to the current and the displacement field strength. The full electric-field tuning of the nonreciprocal transport effect without a magnetic field opens up a new direction for valleytronics in two-dimensional-based devices.
\end{abstract}

DOI: 10.1103/PhysRevResearch.3.033160

\section{INTRODUCTION}

From a symmetry argument, a magnetoelectric anisotropy term in the form of $k \cdot \vec{E} \times \vec{B}$ can generally exist in all systems [1], which gives rise to additional contributions to various magnetoelectric properties in matter [2-5]. Among them, an intriguing subject that has been studied recently is the nonlinear and nonreciprocal transport effect (NRTE). An unusual current-direction dependence on the charge transport is a direct consequence of the NRTE. Phenomenologically, it can be described by a current-dependent resistance of $R=$ $R_{0}[1+\gamma \vec{I} \cdot(\vec{P} \times \vec{B})]$, where $\gamma$ is the NRTE coefficient and $\vec{P}$ is the charge polarization of the system. As is evident from the equation, the NRTE appears only when the current $(\vec{I})$, the magnetic field $(\vec{B})$, and $\vec{P}$ are all nonzero and also perpendicular to each other, setting stringent criteria for the experimental realizations. So far, a number of discoveries for NRTE have been made in various systems, such as bulk-Rashba polar semiconductors [6], chiral magnets [7], noncentrosymmetric superconductors [8-12], and magnetic bilayer heterostructures [13-15]. In theories, the microscopic mechanism of NRTE is typically associated with the velocity asymmetry and the scattering lifetime asymmetry in the Boltzmann transport equation, giving rise to a nonzero transport coefficient in the $E^{2}$ term. Therefore, such a NRTE can be readily probed by

\footnotetext{
*wlee@phys.sinica.edu.tw

Published by the American Physical Society under the terms of the Creative Commons Attribution 4.0 International license. Further distribution of this work must maintain attribution to the author(s) and the published article's title, journal citation, and DOI.
}

measuring the second-harmonic responses to an alternating driving current with a frequency of $\omega$. In general, the nonlinear ( $E^{2}$ term) resistance $\left(R_{2 \omega}\right)$ is negligibly small as compared to $E$-linear resistance $\left(R_{\omega}\right)$, but the ratio of $R_{2 \omega} / R_{\omega}$ can be as large as 0.01 or higher in systems with NRTE.

As it turns out, the Dirac-like systems with chiral fermions are good platforms for the study of NRTE. The trigonal warping effect $[16,17]$ and the chiral property with (pseudo)spin-momentum locking give rise to the asymmetries of energy dispersion and velocity on the Fermi surface. A large current-dependent resistance was reported in a magnetic topological insulator (TI)/TI heterostructure, which was attributed to the Dirac surface states and also magnon-driven asymmetric scattering [14]. On the other hand, in a thin film of nonmagnetic $\mathrm{Bi}_{2} \mathrm{Se}_{3}$, a sizable $R_{2 \omega}$ also appears when the charge transport is dominated by the Dirac surface states [18]. The Dirac Fermi surface with trigonal warping is further distorted by an applied magnetic field parallel to the film surface, giving rise to the observed $R_{2 \omega}$ signals. In other words, the applied magnetic field acts effectively as a spinto-charge converter via the nonlinear resistance channel due to the spin-momentum locking. The aforementioned systems all require an external magnetic field for the appearance of NRTE, which put a severe limitation for the practical device applications. Here, we report a type of NRTE that is rooted in the valley-momentum locking effect of massive and trigonal-warped chiral fermions with Berry phase $2 \pi$ in a dual-gated bilayer graphene (BLG) device. The valley degree of freedom in a BLG is a counterpart of the spin degree of freedom, but the manipulation of the valley polarization, in principle, can be made possible by electric gating only. Large $R_{2 \omega}$ signals were revealed in a dual gate BLG device whenever the Fermi energy $\left(\varepsilon_{\mathrm{F}}\right)$ was tuned to be near the band edges. 

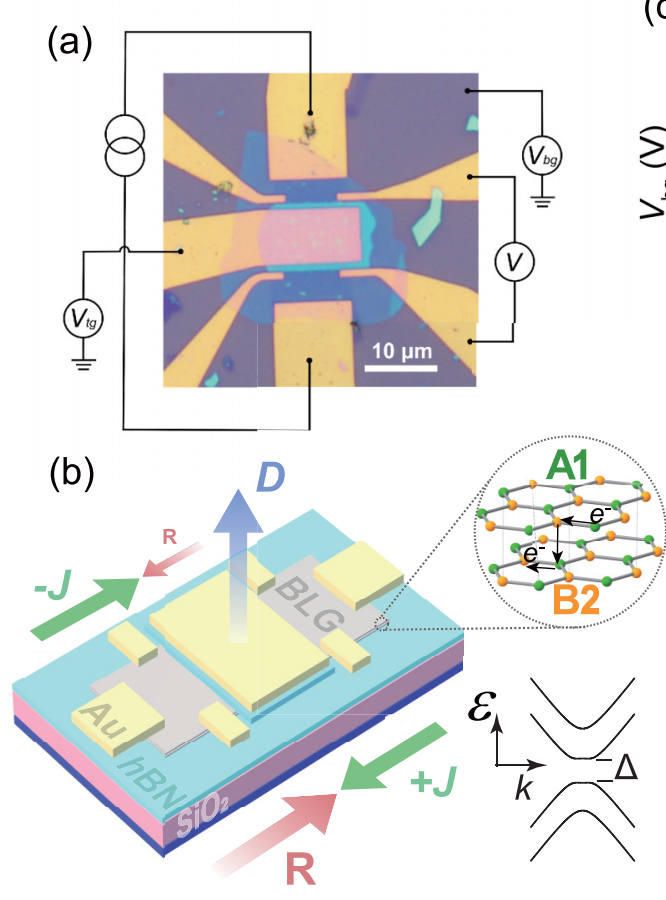
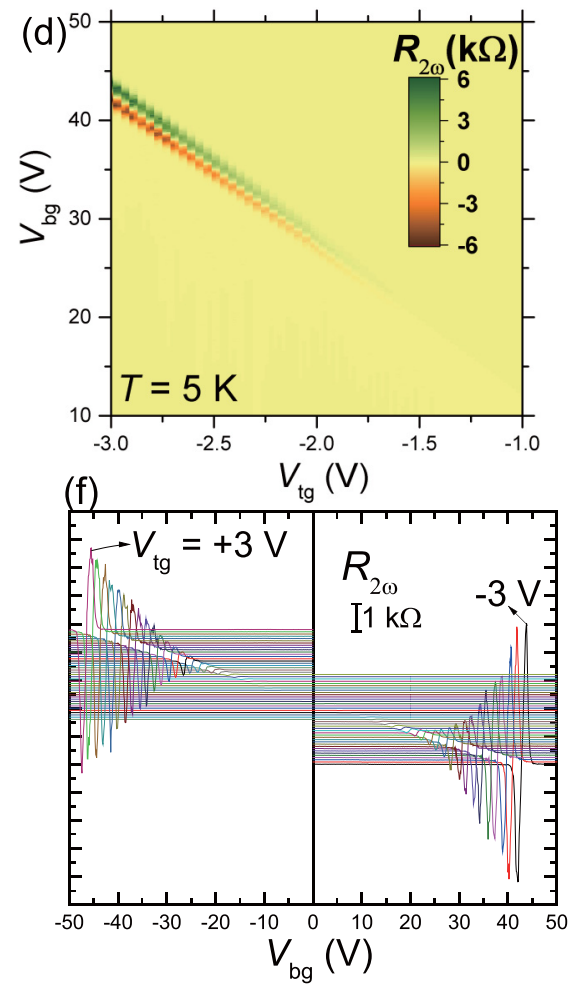

FIG. 1. The setup of a dual-gated BLG device and the measured $R_{\omega}$ and $R_{2 \omega}$ signals. (a) An optical image of a dual-gated BLG device with a schematic for a measurement circuit. (b) An illustration of the current-dependent resistance in a gapped BLG. The inset cartoon shows the AB-stacked BLG, where A1 and B2 are the two sublattices on different layers in a reduced two-component pseudospin representation. Parts (c) and (d) are the $R_{\omega}$ and $R_{2 \omega}$ signals, respectively, as a function of $V_{\mathrm{bg}}$ and $V_{\mathrm{tg}}$ at $T=5 \mathrm{~K}$. The corresponding $R_{\omega}-V_{\mathrm{bg}}$ and $R_{2 \omega}-V_{\mathrm{bg}}$ curves at different $V_{\mathrm{tg}}$ values are shown in (e) and (f), respectively. The curves at different $V_{\mathrm{tg}}$ values are shifted for clarity.

Its magnitude grows larger with increasing displacement field strength $|D|$ and also the bias current. We note that the $|D|$ not only opens up a band gap in a BLG but also effectively polarizes the valley degree of freedom, and thus the exact cancellation of the NRTE from two different valleys of $K+$ and $K-$ is removed to result in a large $R_{2 \omega}$ response. This effect is fully adjustable by electric gating alone and requires no external magnetic field. Those findings can be understood qualitatively from the velocity asymmetry in the Boltzmann transport equation for massive chiral fermions with trigonal warping.

\section{EXPERIMENTAL RESULTS}

Figure 1(a) shows an optical image of a dual-gated BLG device we fabricated, where the measurement circuits were also illustrated (see Appendix A for more information). A BLG was sandwiched between two hexagonal boron nitride (hBN) microcrystals with thicknesses of about a few tens of nanometers, as illustrated in Fig. 1(b). The device geometry was designed to give more than $80 \%$ of the BLG area between the voltage leads to be top-gate effective. Therefore, the measured voltage signals are mostly dominated by the BLG region that can be simultaneously tuned by the top-gate voltage $\left(V_{\mathrm{tg}}\right)$ and bottom-gate voltage $\left(V_{\mathrm{bg}}\right)$, enabling the introduction of a large displacement field $D$ on a BLG. By neglecting the screening effect, the strength of the displacement field can be calculated via $D=\left[\epsilon_{\mathrm{b}}\left(V_{\mathrm{bg}}-V_{\mathrm{bg} 0}\right) / d_{\mathrm{b}}-\epsilon_{\mathrm{t}}\left(V_{\mathrm{tg}}-V_{\mathrm{tg} 0}\right) / d_{\mathrm{t}}\right] / 2$, where $\epsilon_{\mathrm{b}}\left(\epsilon_{\mathrm{t}}\right)$ is the relative dielectric constant for the bottom (top) gate, and $d_{\mathrm{b}}\left(d_{\mathrm{t}}\right)$ is the dielectric layer thickness of the bottom (top) gate. The operation of a dual-gated device not only capacitively varies the density of the system, but it also changes the $D$ values that act on the BLG $[19,20]$. As demonstrated in Fig. 1(b), a band gap $\varepsilon_{g}$ appears due to a finite potential difference $(\Delta)$ between the upper and lower layer of BLG due to $D$, giving rise to a rapid increase of the zero-density sheet resistance $\left(R_{\omega 0}\right)$ with increasing $D$ as shown in Figs. 1(c) and 1(e). The color codes in Fig. 1(c) represent the values of sheet resistance $R_{\omega}$ in logarithmic scale at $T=5 \mathrm{~K}$, and the corresponding $R_{\omega}-V_{\mathrm{bg}}$ curves at different $V_{\mathrm{tg}}$ values ranging from +3 to $-3 \mathrm{~V}$ are shown in Fig. 1(e), where $V_{\mathrm{bg} 0}$ and $V_{\operatorname{tg} 0}$ were determined to be $\approx-1.86$ and $-0.15 \mathrm{~V}$, respectively. The $R_{\omega 0}$ equals about $2.2 \mathrm{k} \Omega$ at zero $V_{\mathrm{tg}}$, and it increases by more than two orders of magnitude of $R_{\omega} \approx 285.6 \mathrm{k} \Omega$ at $V_{\mathrm{tg}}=-3 \mathrm{~V}$, which corresponds to $D \approx$ $+0.58 \mathrm{~V} / \mathrm{nm}$. The large variation in $R_{\omega}$ indicates a high device quality, which is also supported by the high Hall mobility of $\approx 10000 \mathrm{~cm}^{2} / \mathrm{V}$ s as determined from the Hall measurements (see Appendix B). Surprisingly, large second-harmonic signals $R_{2 \omega}$ were uncovered and shown in Figs. 1(d) and 1(f). The color codes in Fig. 1(d) indicate the $R_{2 \omega}$ values in linear scale, and the corresponding $R_{2 \omega}-V_{\mathrm{bg}}$ curves at different $V_{\mathrm{tg}}$ values are shown in Fig. 1(f). Pronounced $R_{2 \omega}$ values appear near the zero-density regime with magnitudes rapidly growing for $\left|V_{\mathrm{tg}}\right| \geqslant 2 \mathrm{~V}$. As $V_{\mathrm{bg}}$ sweeps from $+50 \mathrm{~V}$ to $-50 \mathrm{~V}, R_{2 \omega}$ first reaches a local maximum and then rapidly falls off to 
(a)

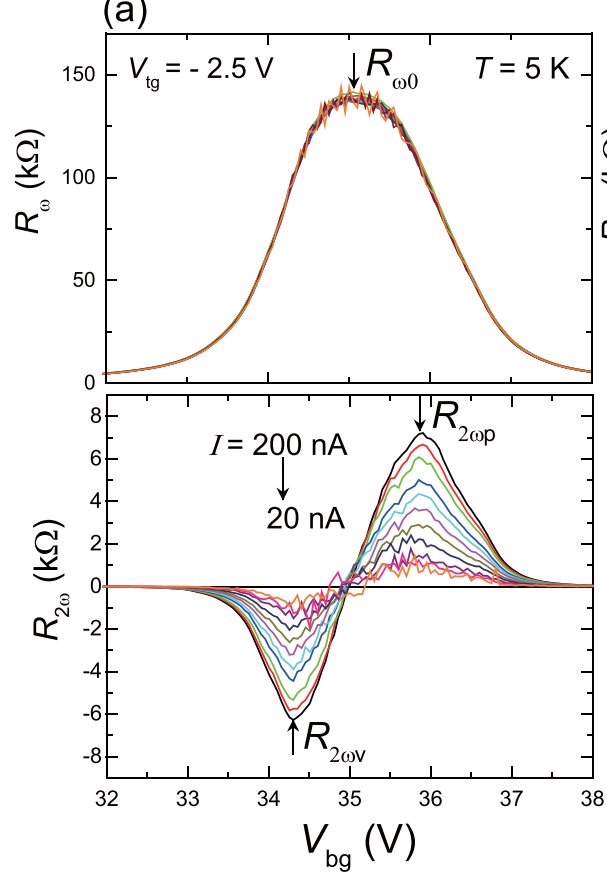

(b)

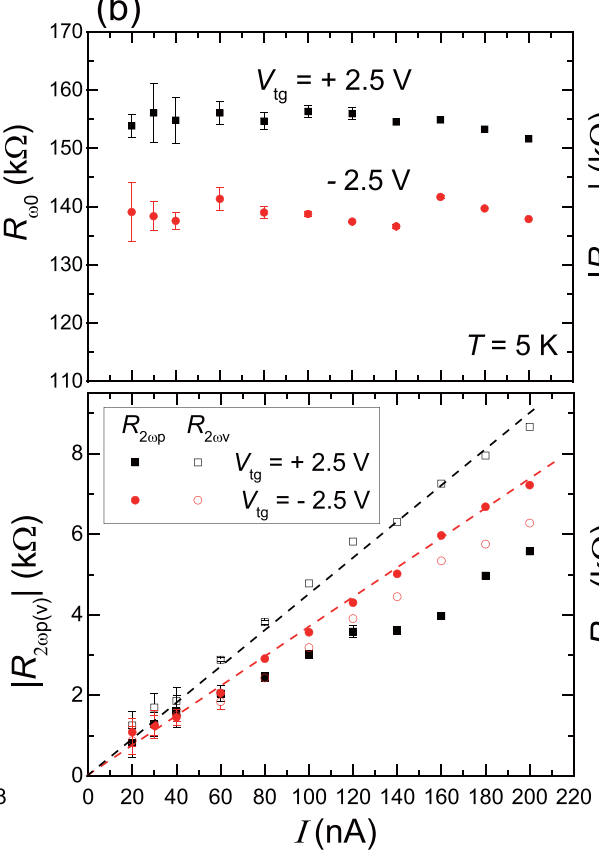

(c)

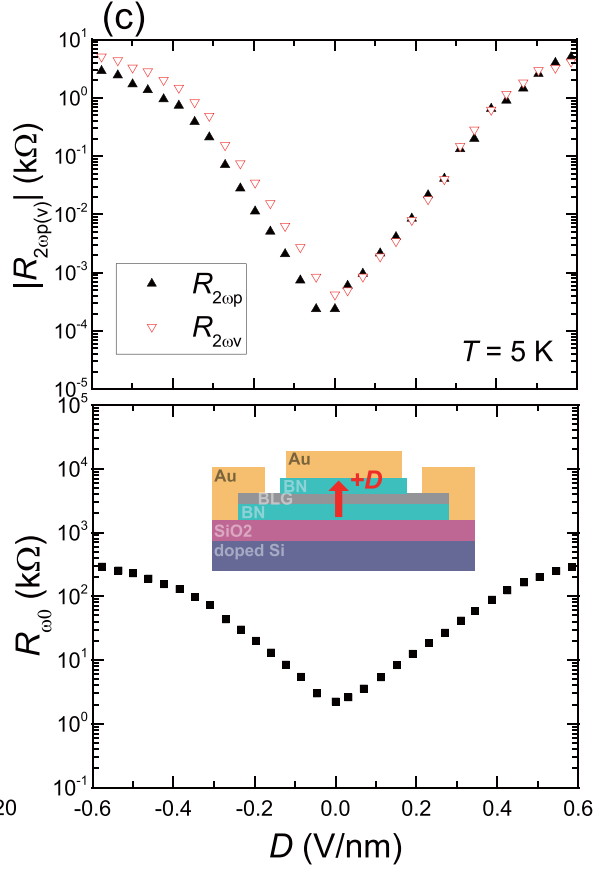

FIG. 2. The current (I)-dependence and $D$-dependence of the NRTE. (a) The upper panel and lower panel show the $R_{\omega}-V_{\mathrm{bg}}$ and $R_{2 \omega}-V_{\mathrm{bg}}$ curves, respectively, with different bias currents ranging from 20 to $200 \mathrm{nA}$ at $T=5 \mathrm{~K}$. The local extremal values of $R_{2 \omega}\left(R_{2 \omega \mathrm{p}}\right.$ and $\left.R_{2 \omega \mathrm{v}}\right)$ are practically linear with $I$ [lower panel of (b)], which is in big contrast to the nearly $I$-independent $R_{\omega 0}$ [upper panel of (b)]. The corresponding $D$-dependence of local extremal values of $R_{2 \omega}$ and $R_{\omega}$ is plotted in the upper and lower panel, respectively, in (c). The inset cartoon of the lower panel illustrates the cross-sectional view of a dual-gated device with a given displacement field $D$.

a local minimum with an opposite sign. At $V_{\mathrm{tg}}=-3 \mathrm{~V}$, the magnitude of $R_{2 \omega}$ is as large as $5.0 \mathrm{k} \Omega$. The bias current $I$ equals $100 \mathrm{nA}$ for all the data shown in Fig. 1, and the curves at different $V_{\text {tg }}$ values in Figs. 1(e) and 1(f) are shifted for clarity.

The current dependence of $R_{\omega}$ and $R_{2 \omega}$ with $V_{\mathrm{tg}}=-2.5 \mathrm{~V}$ and $T=5 \mathrm{~K}$ is shown in the upper panel and lower panel, respectively, of Fig. 2(a). By setting different $I$ values ranging from 20 to $200 \mathrm{nA}$, the local extremal values in $R_{2 \omega}-V_{\mathrm{bg}}$ curves increase in magnitude with increasing $I$, where $R_{2 \omega \mathrm{p}}$ and $R_{2 \omega \mathrm{v}}$ are defined as the values at the peak and valley, respectively, of the $R_{2 \omega}-V_{\mathrm{bg}}$ curve as shown in the lower panel of Fig. 2(a). On the contrary, the $R_{\omega}$ data in the upper panel of Fig. 2(a) show negligible variations with $I$, where $R_{\omega 0}$ is defined as the peak value in an $R_{\omega}-V_{\mathrm{bg}}$ curve. The nearly $I$-independent $R_{\omega 0}$ thus suggests an insignificant Joule heating effect during the measurements with a current up to $200 \mathrm{nA}$. The detailed $I$-dependence of $R_{\omega 0}$ and the magnitude of $R_{2 \omega \mathrm{p}, \mathrm{v}}$ are plotted in the upper and lower panels of Fig. 2(b), respectively. For a 10 -fold increase in $I$, the variation of $R_{\omega 0}$ at $V_{\mathrm{tg}}=-2.5 \mathrm{~V}$ [solid circles in the upper panel of Fig. 2(b)] is less than $\approx 3 \%$, which is in large contrast with the practical $I$-linear dependence of $\left|R_{2 \omega \mathrm{p}(\mathrm{v})}\right|$ as shown in the lower panel of Fig. 2(b). The $R_{2 \omega \mathrm{p}}$ at $V_{\mathrm{tg}}=-2.5 \mathrm{~V}$ increases from a value of $1.08 \mathrm{k} \Omega$ for $I=20 \mathrm{nA}$ up to $7.22 \mathrm{k} \Omega$ for $I=200 \mathrm{nA}$. $R_{2 \omega \mathrm{v}}$ [open circles in the lower panel of Fig. 2(b)], on the other hand, shows a consistent behavior with a slight difference in magnitude. Upon reversing the sign of $V_{\mathrm{tg}}$, similar behaviors were observed for $V_{\mathrm{tg}}=+2.5 \mathrm{~V}$. As revealed in Fig. 1(f), we remark that the same $R_{2 \omega}-V_{\mathrm{bg}}$ profiles as that in the lower panel of Fig. 2(a) are found, regardless of the sign of the $V_{\mathrm{tg}}$ and thus $D$. By collecting all the data with different $V_{\mathrm{tg}}$ values, the $D$-dependence of $\left|R_{2 \omega \mathrm{p}(\mathrm{v})}\right|$ and $R_{\omega 0}$ in logarithmic scales is shown in the upper panel and lower panel, respectively, of Fig. 2(c). Both $\left|R_{2 \omega \mathrm{p}(\mathrm{v})}\right|$ and $R_{\omega 0}$ are exponentially growing with $D$ in the low- $D$ regime, and it slows down for $|D| \geqslant$ $0.4 \mathrm{~V} / \mathrm{nm}$. By tuning $D$ up to about a magnitude of $\approx 0.58$ $\mathrm{V} / \mathrm{nm}$, we remark that the $\left|R_{2 \omega \mathrm{p}(\mathrm{v})}\right|$ spans more than four orders of magnitude, which is more than twofold larger than that for $R_{\omega 0}$. The inset cartoon in the lower panel of Fig. 2(c) illustrates the sign of $D$ in relation to the device geometry.

In a dual-gated BLG device, the unscreened $D$ values as a function of $V_{\mathrm{bg}}$ and $V_{\mathrm{tg}}$ can be calculated as shown in Fig. 3(a). The sweeping of gate voltages along a $D$-constant contour line ensures the shifting of only the $\varepsilon_{\mathrm{F}}$ in BLG while keeping the degree of inversion asymmetry unchanged. As an example, a sweeping along a black line, connecting point 1 and point 2 in Fig. 3(a) along a $D$-constant contour line, will result in a shifting of $\varepsilon_{\mathrm{F}}$ from the conduction band to the valence band as illustrated in the lower panel of Fig. 3(a). We carried out such sweepings along $D$-constant contour lines at 10 different $D$ values ranging from +0.5 to $-0.5 \mathrm{~V} / \mathrm{nm}$. The upper and lower panels of Fig. 3(b) show the measurement results for $R_{\omega}$ and $\left|R_{2 \omega}\right|$, respectively, as a function of the corresponding sheet density $n_{2 \mathrm{D}}$. When $\varepsilon_{\mathrm{F}}=0\left(n_{2 \mathrm{D}}=0\right), R_{\omega}$ attains a maximum value of $R_{\omega 0}$, but $R_{2 \omega}$ is nearly zero. As $\varepsilon_{\mathrm{F}}$ moves toward the conduction (valence) band with $n_{2 \mathrm{D}} \leqslant 0\left(n_{2 \mathrm{D}} \geqslant 0\right),\left|R_{2 \omega}\right|$ first increases rapidly to a local maximum and then falls off to a vanishingly small value for $\left|n_{2 \mathrm{D}}\right| \geqslant 3 \times 10^{11} \mathrm{~cm}^{-2} . \Delta n_{2 \mathrm{D}}$ is the sheet density at which $\left|R_{2 \omega}\right|$ reached a maximum, as illustrated in the lower panel of Fig. 3(b). An enlarged view of the $R_{2 \omega}-n_{2 \mathrm{D}}$ data in a linear scale is shown in Fig. 3(c), 
(a)

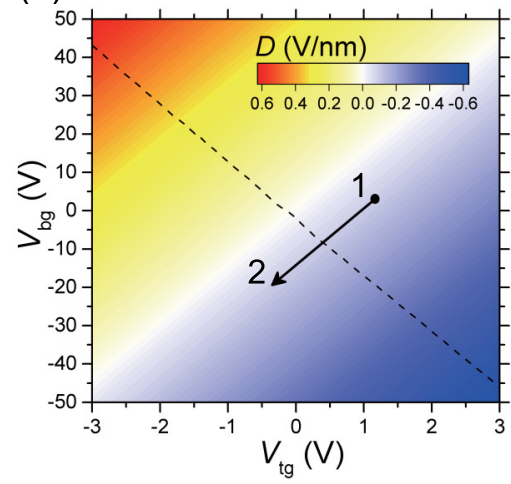

varying $\varepsilon_{\mathrm{F}}$ with a constant $D$

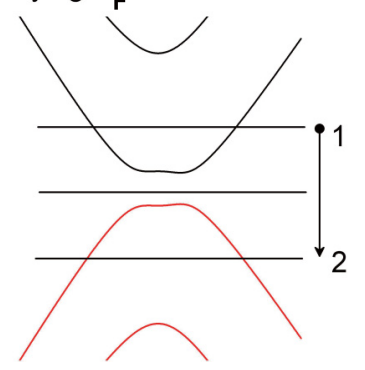

(b) $\underline{D(V / n m)}$

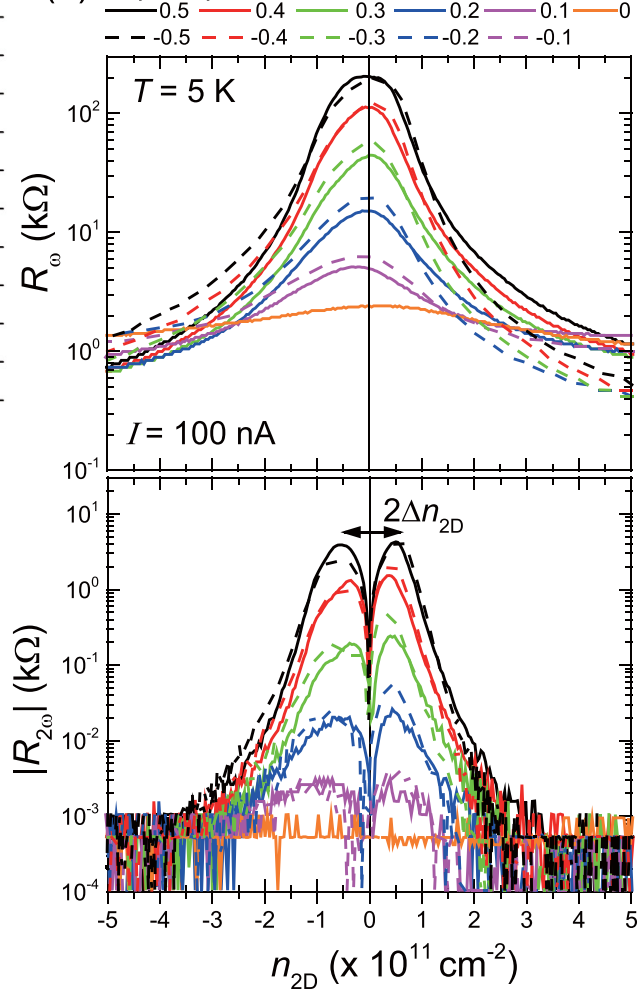

(c)
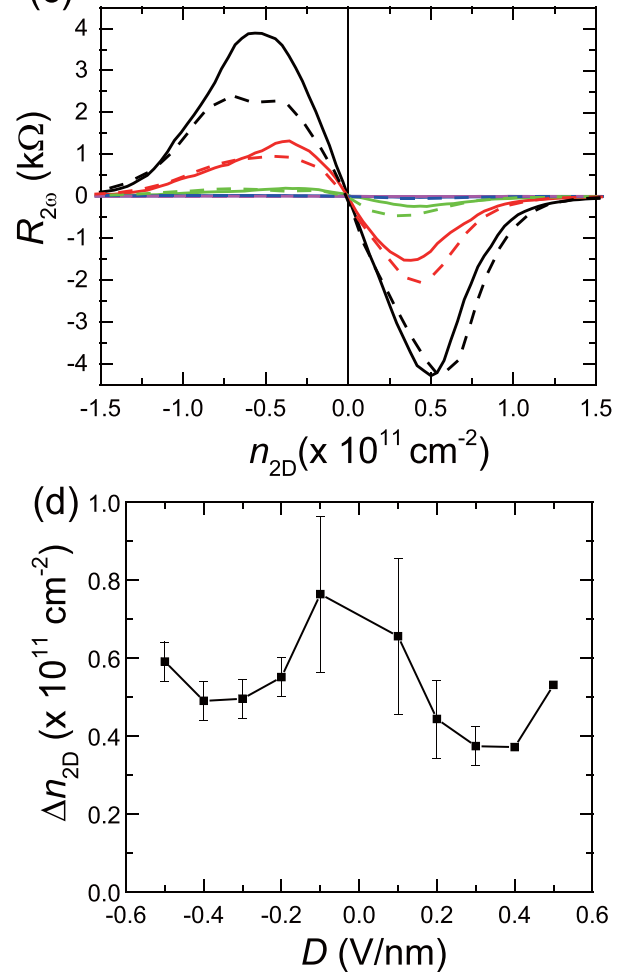

FIG. 3. The tuning of Fermi energy $\left(\varepsilon_{\mathrm{F}}\right)$ with a constant $D$. (a) The $D$ values vs $V_{\mathrm{bg}}$ and $V_{\mathrm{tg}}$. A $D$-constant sweep from point 1 to point 2 in (a) corresponds to a shift in $\varepsilon_{\mathrm{F}}$ from the conduction band to the valence band across a band gap. (b) The $R_{\omega}$ and the magnitude of $R_{2 \omega}\left(\left|R_{2 \omega}\right|\right)$ in logarithmic scales are plotted as a function of the sheet density $n_{2 \mathrm{D}}$ with various $D$ values ranging from +0.5 to $-0.5 \mathrm{~V} / \mathrm{nm}$. The $\Delta n_{2 \mathrm{D}}$, as defined in the lower panel of (b), is the sheet density at which $R_{2 \omega}$ attains a local extremal value. Part (c) shows an enlarged view of $R_{2 \omega}-n_{2 \mathrm{D}}$, which shares the same color codes and lines denoted in (b). The corresponding $D$-dependence of $\Delta n_{2 \mathrm{D}}$ is shown in (d).

which shares the same color codes as in Fig. 3(b). As noted before, by reversing the sign of $D$ [dashed lines in Figs. 3(b) and 3(c)], consistent behaviors of $R_{\omega}$ and $\left|R_{2 \omega}\right|$ are observed with a minor difference in magnitude, but no sign changes in $R_{2 \omega}$ were found due to the sign reversal of $D$. The extracted $\Delta n_{2 \mathrm{D}}$ is plotted as a function of $D$, as shown in Fig. 3(d). For $|D| \leqslant 0.4 \mathrm{~V} / \mathrm{nm}, \Delta n_{2 \mathrm{D}}$ tends to drop slightly with increasing $|D|$ to a value of $\approx 3.7 \times 10^{10} \mathrm{~cm}^{-2}$ at $D=0.4 \mathrm{~V} / \mathrm{nm}$.

The $T$-dependent measurements were carried out to extract the band gap $\varepsilon_{\mathrm{g}}$ using a thermal-activation model for the conductance. The normalized zero-density conductance of $R_{\omega 0}(D=0) / R_{\omega 0}$ versus $1 / T$ curves for different $D$ values up to $\approx 0.58 \mathrm{~V} / \mathrm{nm}$ is plotted in Fig. 4(a), where the red lines are fitting curves using a formula of $R_{\omega 0}(D=0) / R_{\omega 0}=$ $A \exp \left[-\varepsilon_{\mathrm{g}} / 2 k_{B} T\right]+C$. The extracted band gap $\varepsilon_{\mathrm{g}}$ as a function of the corresponding $D$ is shown in Fig. 4(b), and $\varepsilon_{\mathrm{g}}$ is practically linear with $D$, giving $\varepsilon_{\mathrm{g}} \approx 60 \mathrm{meV}$ at $D=+0.58$ $\mathrm{V} / \mathrm{nm}$. The NRTE turns out to diminish faster with increasing temperature as compared to $R_{\omega 0}$. Figure 4 (c) shows the $T$-dependence of the ratio for local extremal $R_{2 \omega}$ to the corresponding $R_{\omega}$, where it decays from a value of about $2.8 \%$ at $T=5 \mathrm{~K}$ to $0.28 \%$ at $T=80 \mathrm{~K}$. The $\Delta V_{\mathrm{bg}}$, defined as the $V_{\mathrm{bg}}$ difference between the local extremes in $R_{2 \omega}-V_{\mathrm{bg}}$ curves, is shown in Fig. 4(d), which shows similar behavior to $\Delta n_{2 \mathrm{D}}$ in Fig. 3(d). At $T=5 \mathrm{~K}, \Delta V_{\text {bg }}$ first decreases with increasing $|D|$ up to a critical value of $D_{\mathrm{c}} \approx 0.4 \mathrm{~V} / \mathrm{nm}$. For $D \geqslant D_{\mathrm{c}}, \Delta V_{\mathrm{bg}}$ shows a small increase with $|D|$. We note that $D_{\mathrm{c}}$ turns out to move to a higher value as $T$ increases. For all the $T$-dependent data, the $R_{2 \omega \mathrm{p}(\mathrm{v})}$ versus the corresponding $R_{\omega}$ collapsed on a single curve, as demonstrated in Fig. 4(e), giving a relation of $R_{2 \omega \mathrm{p}(\mathrm{v})} \propto R_{\omega}^{2.02}$.

\section{NUMERICAL CALCULATIONS OF NRTE}

As illustrated in the lower panel of Fig. 5(a), the sublattices $\mathrm{A} 1$ and B2 on different layers form an effective honeycomb lattice in the reduced two-component pseudospin representation with two different valleys of $K+$ and $K-$. The reduced $2 \times 2$ Hamiltonian of a BLG [21] can be described by $\mathcal{H}_{\mathrm{BLG}}=$ $-\frac{\hbar^{2}}{2 m^{*}} \sigma \cdot \vec{\kappa}^{2}$, where $\vec{\kappa}^{2} \equiv\left[\left(k_{x}^{2}-k_{y}^{2}\right) \hat{x}+2 k_{x} k_{y} \hat{y}\right], m^{*}$ is the effective mass, $\sigma$ is the Pauli spin matrices, and $\hat{x}(\hat{y})$ is a unit vector alone the $x(y)$ axis. The resulting two-component pseudospin states, referring to the electron probabilities on sublattices A1 and B2, are thus chiral fermions with the valley-momentum locking effect. By further considering trigonal warping and an asymmetry parameter $\Delta$ [22], the energy dispersion can be calculated and expressed as

$$
\begin{aligned}
\varepsilon_{\alpha, \xi}^{2}(p, \phi)= & \frac{\gamma_{1}^{2}}{2}+\frac{\Delta^{2}}{4}+\left(v^{2}+\frac{v_{3}^{2}}{2}\right) p^{2}+(-1)^{\alpha} \sqrt{\Gamma}, \quad \text { and } \\
\Gamma= & \frac{1}{4}\left(\gamma_{1}^{2}-v_{3}^{2} p^{2}\right)^{2}+v^{2} p^{2}\left[\gamma_{1}^{2}+\Delta^{2}+v_{3}^{2} p^{2}\right] \\
& +2 \xi \gamma_{1} v_{3} v^{2} p^{3} \cos (3 \phi),
\end{aligned}
$$


(a)
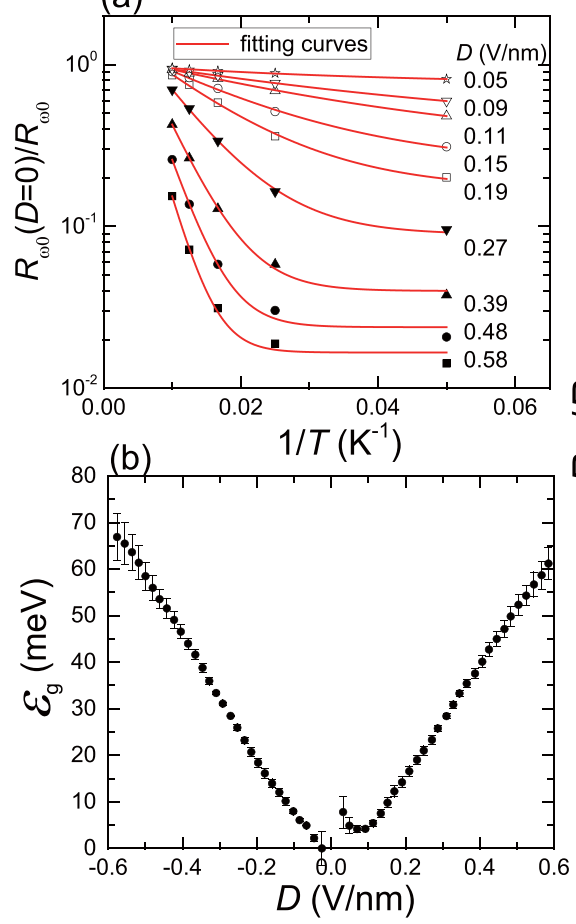

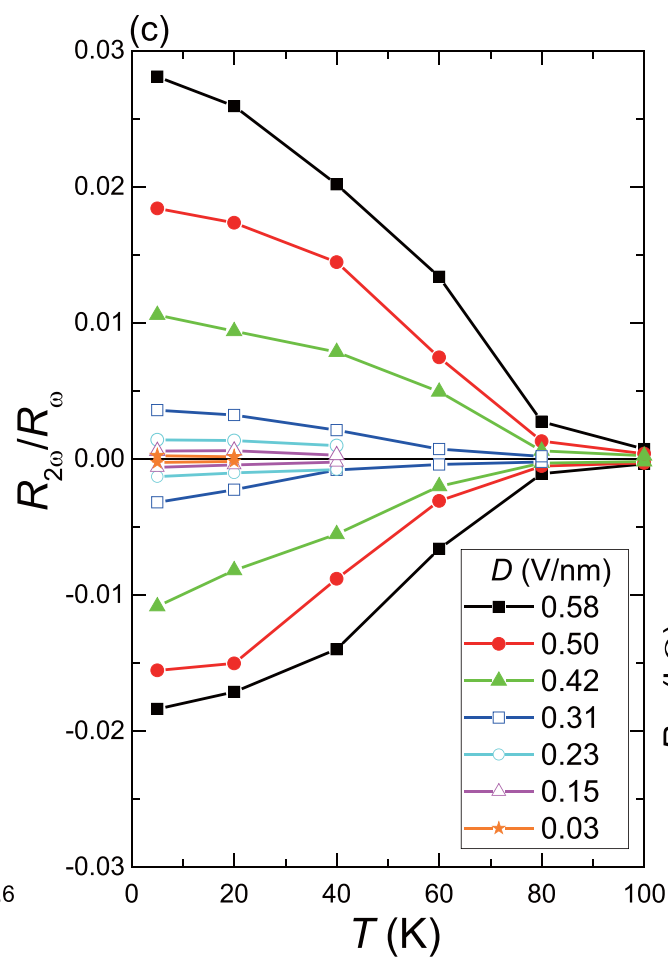

(d)

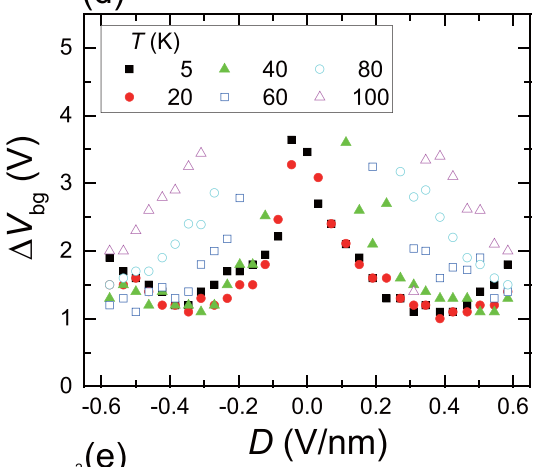

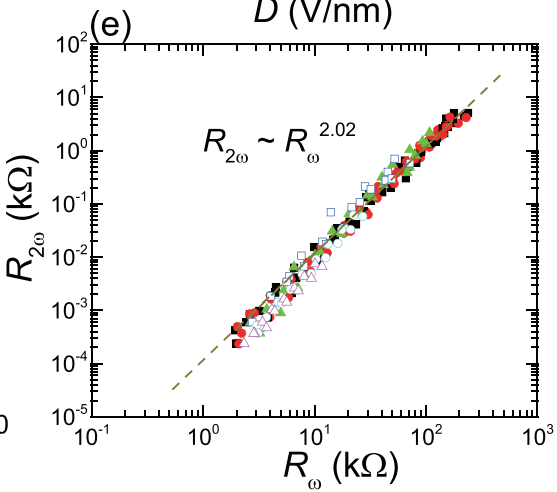

FIG. 4. The temperature dependence of $R_{\omega}$ and $R_{2 \omega}$. (a) Normalized sheet conductance $\left[R_{\omega 0}(D=0) / R_{\omega 0}\right]$ as a function of $1 / T$ at different $D$ values. The red lines are the fitting curves using the thermal-activated conductivity formula. The extracted band gap $\varepsilon_{\mathrm{g}}$ is nearly linear with $D$ as shown in (b), giving a band gap of $\varepsilon_{\mathrm{g}} \approx 67 \mathrm{meV}$ at $D=0.58 \mathrm{~V} / \mathrm{nm}$. (c) The ratio of local extremal $R_{2 \omega}$ to the corresponding $R_{\omega}$ as a function of $T$ for different $D$ values. The $\Delta V_{\mathrm{bg}}$ values are obtained from the difference of the $V_{\mathrm{bg}}$ values at which the $R_{2 \omega}$ attains local extremal values in each $R_{2 \omega}-V_{\mathrm{bg}}$ curve. At $T=5 \mathrm{~K}, \Delta V_{\mathrm{bg}}$ first drops with increasing $D$ up to a turning point of $D_{\mathrm{c}} \approx 0.4 \mathrm{~V} / \mathrm{nm}$, and then it starts to increase in the high- $D$ regime. $D_{\mathrm{c}}$ turns out to shift to a bigger value with increasing $T$ as shown in (d). (e) The local extremal $R_{2 \omega}$ values as a function of the corresponding $R_{\omega}$ in logarithmic scales, giving a relation of $R_{2 \omega} \propto R_{\omega}^{2.02}$. The data points share the same symbol and color code as that in (d).

where $(p=\hbar k, \phi)$ is the momentum in polar coordinate, $\gamma_{1}$ is the interlayer nearest-neighbor hopping amplitude, $\xi=$ $+1(-1)$ refers to the $K+(K-)$ valley, and $v$ is the Fermi velocity. The trigonal warping derives from the additional velocity term of $v_{3} \equiv \sqrt{3} a \gamma_{3} /(2 \hbar)$, where $a$ is the lattice parameter for the sublattice and $\gamma_{3}$ is the next-nearest interlayer hopping amplitude as illustrated in the inset cartoon of Fig. 1(b). As revealed from Eq. (1), there are four bands as shown in the upper panel of Fig. 5(a) for $\Delta=100 \mathrm{meV}$, where the color codes represent the energy. The black and red contours for the lower energy band $(\alpha=1)$ are at $\varepsilon=+300$ and $+100 \mathrm{meV}$, respectively, where the corresponding constant energy contours as a function of $k_{\mathrm{x}}$ and $k_{\mathrm{y}}$ are shown in Fig. 5(b). Apparent distortions of the Fermi surface from symmetric circles (dashed lines) are observed, and we note that the distorted Fermi surfaces resemble rounded triangles pointing to the opposite direction for $K+$ and $K-$. Similar behaviors are found for $\varepsilon=-300$ and $-100 \mathrm{meV}$ as shown as green and blue contours in Fig. 5(c). The calculated velocity $\left(\partial \varepsilon / \partial k_{\mathrm{x}}\right)$ and $\partial^{2} \varepsilon / \partial k_{\mathrm{x}}^{2}$ as a function of $k_{\mathrm{x}}$ for $\Delta=100 \mathrm{meV}$ are shown in the upper and lower panels of Fig. 5(d), respectively, for lower energy bands of $K+$ and $K-$ valleys. For each valley, there are asymmetries in both $\partial \varepsilon / \partial k_{\mathrm{x}}$ and $\partial^{2} \varepsilon / \partial k_{\mathrm{x}}^{2}$ with respect to $k_{\mathrm{x}}$, but it cancels exactly while summing up contributions from both valleys of $K+$ and $K-$. Therefore, an additional breaking of the valley symmetry (i.e., valley polarization) is needed in order to have a nonzero contribution due to the asymmetry terms of $\partial \varepsilon / \partial k_{\mathrm{x}}$ and $\partial^{2} \varepsilon / \partial k_{\mathrm{x}}^{2}$, which is required for the appearance of NRTE to be shown as follows.

Based on the Boltzmann transport theorem [23], the nonequilibrium distribution function $\left(g_{\mathrm{k}}\right)$ up to the second order of driving electric-field $\left(E^{2}\right)$ can be described by $g_{\mathrm{k}}=$ $\frac{e \tau E_{\mathrm{x}}}{\hbar} \frac{\partial f_{0}}{\partial k_{\mathrm{x}}}+\left(\frac{e \tau E_{\mathrm{x}}}{\hbar}\right)^{2} \frac{\partial^{2} f_{0}}{\partial k_{\mathrm{x}}^{2}}$, where we used $\vec{E}=E_{\mathrm{x}} \hat{i}$. The resulting second-harmonic conductance $\sigma_{2 \omega}$ due to NRTE at $T=0 \mathrm{~K}$ for the low-energy bands $(\alpha=1)$ can be expressed as (see Appendix C)

$$
\begin{aligned}
\sigma_{2 \omega, \xi}(\varepsilon)= & \frac{q^{3}}{2 \pi \hbar^{2}} \int d \phi \tau^{2} p \cos ^{3} \\
& \times\left.\phi\left[3 \frac{\left|v_{\mathrm{k}, \xi}\right|}{v_{\mathrm{k}, \xi}} \frac{\partial v_{\mathrm{k}, \xi}}{\partial p}-\frac{\partial\left|v_{\mathrm{k}, \xi}\right|}{\partial p}\right]\right|_{\varepsilon},
\end{aligned}
$$

where $q$ is the charge of carriers, $\tau$ is the transport lifetime, $p=\hbar k$, and $v_{\mathrm{k}, \xi}=\partial \varepsilon_{1, \xi} / \partial p$. For simplicity, we neglect the complex asymmetric scattering effects for chiral fermions in a BLG, and Eq. (2) reduces to a simple form of $\sigma_{2 \omega, \xi}=$ $\frac{q^{3} \tau^{2}}{2 \pi \hbar^{2}} I_{2 \omega, \xi}$, where the $\varepsilon_{\xi}$-dependent $I_{2 \omega, \xi}$ term with a unit of velocity $\left(\mathrm{s}^{-1} \mathrm{~m}\right)$ can be numerically calculated from Eq. (1) and shown in Fig. 6(a) for $\Delta=50 \mathrm{meV}$ (see Appendix C). For $\xi=+1(K+$ valley $)$, as $\varepsilon$ decreases from $55 \mathrm{meV}$, the magnitude of $I_{2 \omega,+1}$ increases, reaching a maximum value near the 
(a)

\section{Calulated band structure} with trigonal warping
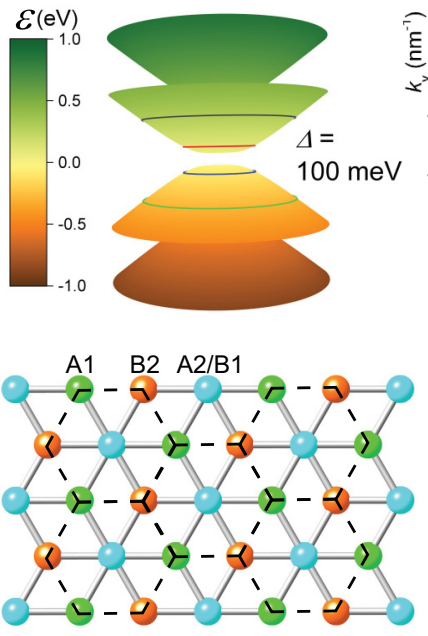
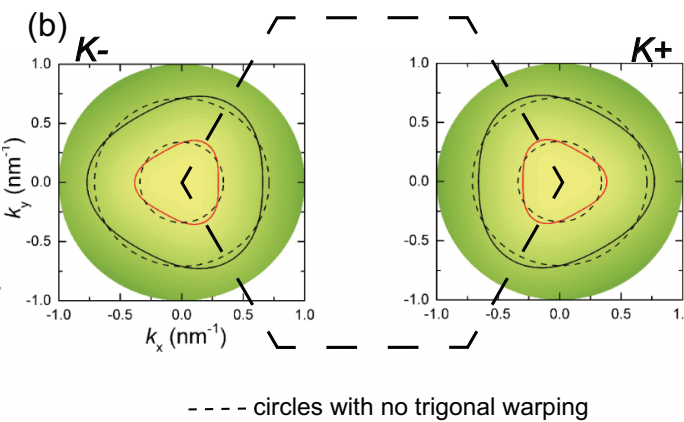

$K+$

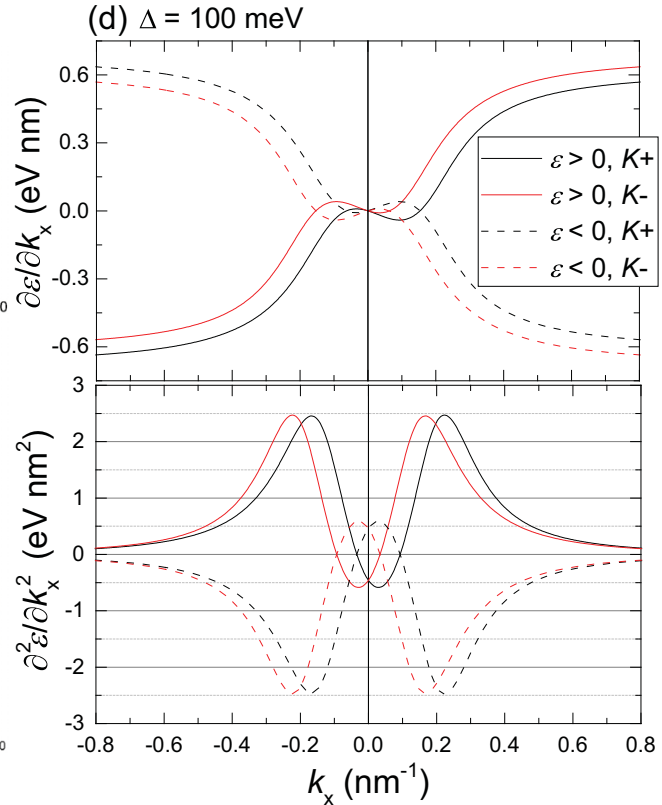

FIG. 5. Calculated band structures and velocity asymmetry of a gapped BLG with trigonal warping. (a) The band structure of a BLG with asymmetric parameter $\Delta=100 \mathrm{meV}$. The lower panel of (a) shows the A1 and B2 sublattices in a reduced honeycomb lattice depicted by black dashed lines, comprising the two different valleys of $K+$ and $K-$. The corresponding constant energy contour plots of the low-energy band for $K+$ and $K-$ are shown as solid lines in the right panel and left panel of (b), respectively. Part (c) shows the results for the negative low-energy band. The color codes for the solid lines in (b) and (c) are the same as that in (a), indicating Fermi surfaces at different energies. Those Fermi surfaces show apparent deviations from a perfect circle [dashed lines in (b) and (c)] due to the trigonal warping effect, which thus gives rise to the asymmetries in velocity $\left(\partial \varepsilon / \partial k_{\mathrm{x}}\right)$ and $\partial^{2} \varepsilon / \partial k_{\mathrm{x}}^{2}$ at Fermi surfaces as demonstrated in the upper panel and lower panel of (d), respectively.

band edge at $\varepsilon \approx 25 \mathrm{meV}$ [dashed line in Fig. 6(a)]. For $\varepsilon \leqslant$ $25 \mathrm{meV}$, a Lifshitz transition occurs [21,24,25], and the single rounded-triangle Fermi surface transformed into multiple separated ellipses as illustrated in the right panel of Fig. 6(a) (see Appendix C). The magnitude of $I_{2 \omega,+1}$ drops rapidly to zero for $\varepsilon \leqslant 24.3 \mathrm{meV}$, where the total area of ellipses and thus the density of states are negligibly small. As expected, the $I_{2 \omega,+1}$ turns out to be canceled exactly by $I_{2 \omega,-1}$ for $K-$ at the same $\varepsilon$ [red line in Fig. 6(a)]. We remark that such a Lifshitz transition is a direct consequence of the trigonal warping effect that becomes significant at lower energy, and it happens for all $\Delta$ values near the band edges [25]. The corresponding energy $\varepsilon_{\mathrm{L}}$ for the occurrence of the Lifshitz transition equals $\approx 1 \mathrm{meV}$ for $D=0$, and $\varepsilon_{\mathrm{L}} \approx \Delta / 2$ for a finite $D$. For more rigorous calculations, the asymmetric scatterings in $\tau$ and the correlation effect should be considered [14,26,27], which is beyond the scope of the current work. However, we argue that the inclusion of the asymmetric scatterings in $\tau$ should further amplify the asymmetry terms in Eq. (2) and thus may not affect the qualitative scenario that we described here.

\section{DISCUSSIONS}

The breaking of the inversion symmetry by $D$ not only results in a finite $\varepsilon_{\mathrm{g}}$ but also gives rise to a valley polarization in a gapped BLG $[28,29]$. A large nonlocal resistance has been reported in a gapped BLG and is attributed to the inverse valley Hall effect with $D$-enhanced valley polarization [30-32]. As illustrated in Fig. 6(b), the $D$-induced potential difference between the upper and lower layer of a BLG causes a small energy difference for the electrons in different sublattices (valleys). The energy difference can be described by $\Delta \varepsilon \approx q D d \delta n / n_{\mathrm{c}}$, where $d$ is the distance between layers, $n_{\mathrm{c}} \equiv \frac{\gamma_{1}^{2}}{\pi \hbar^{2} v^{2}}$, and $\delta n$ is the $D$-induced density difference between layers. The resulting Fermi energies in $K+$ (black solid line) and $K-$ (red solid line) are then shifted in the opposite direction, as illustrated in the right panel of Fig. 6(b). The total second-harmonic conductance is then described by

$$
\begin{aligned}
\sigma_{2 \omega} & =\sigma_{2 \omega,+1}\left(\varepsilon_{\mathrm{F}}+\Delta \varepsilon\right)+\sigma_{2 \omega,-1}\left(\varepsilon_{\mathrm{F}}-\Delta \varepsilon\right) \\
& =\frac{q^{3} \tau^{2}}{\pi \hbar^{2}}\left[\frac{\partial I_{2 \omega,+1}}{\partial \varepsilon}\right] \Delta \varepsilon .
\end{aligned}
$$

As a result, a nonzero $\sigma_{2 \omega}$ appears when $\Delta \varepsilon$ and thus $D$ is finite. The measured second-harmonic resistance can be linked to the calculated $\sigma_{2 \omega}$ via $R_{2 \omega}=-\frac{I_{0}}{W} \frac{\sigma_{2}}{\sigma^{3}}$, where $W$ is the width of the device, $I_{0}$ is the magnitude of the bias current, and $\sigma_{\omega}=1 / R_{\omega}$ (see Appendix C). It thus justified the $I$-linear dependence shown in the lower panel of Fig. 2(b). The calculated $\sigma_{2 \omega}$ then reflects the behavior of $R_{2 \omega}$. The numerical calculations of the $\partial I_{2 \omega,+1} / \partial \varepsilon$ term for the $K+$ valley are plotted as a function of shifted energy $(\varepsilon-\Delta / 2)$ shown in Fig. 6(c) for three different $\Delta$ values of 10, 30, and $50 \mathrm{meV}$. For the $K-$ valley, it gives the same values with the opposite sign. The shaded area corresponds to a small energy window $(\approx 0.2 \mathrm{meV})$ in which the Lifshitz transition occurs, where large and discontinuous values of $\partial I_{2 \omega,+1} / \partial \varepsilon$ are found due to the abrupt changes in the density of states 


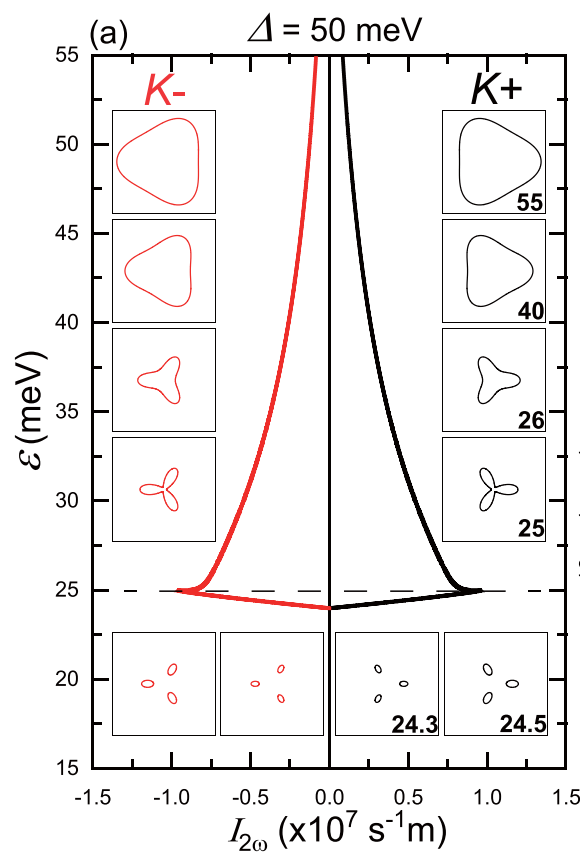

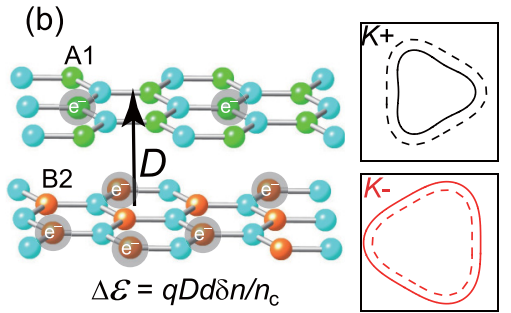

(c)

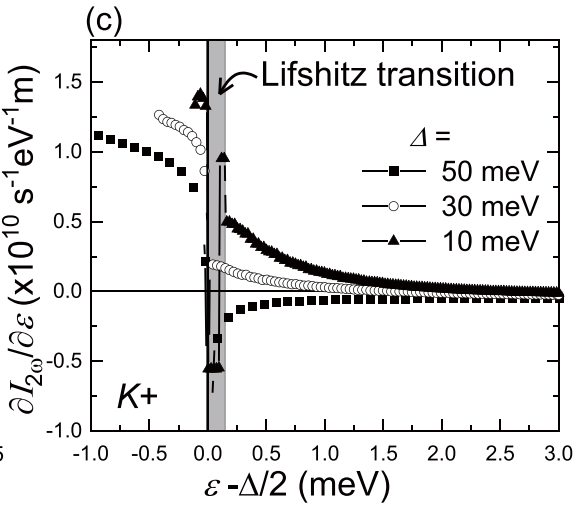

(d) eigen pseudospin states
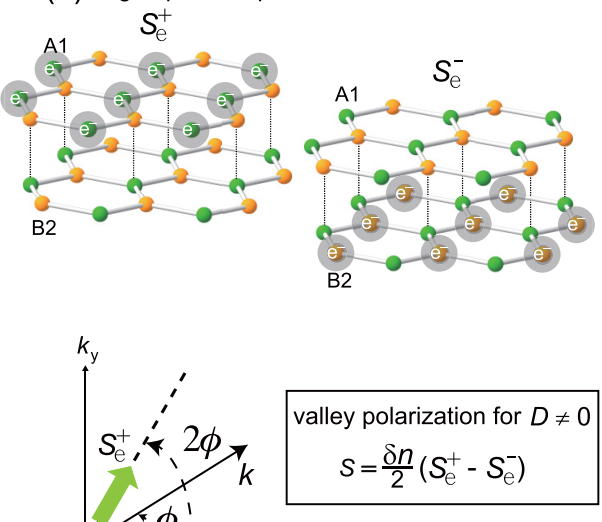

FIG. 6. Numerical calculations for the nonreciprocal term of $\sigma_{2 \omega}=\frac{q^{3} \tau^{2}}{2 \pi \hbar^{2}} I_{2 \omega}$ in the Boltzmann transport equation. Part (a) plots the energy dependence of the calculated $I_{2 \omega}$ with $\Delta=50 \mathrm{meV}$. The black and red denote $K+$ and $K-$, respectively. The six insets on the right (left) of (a) correspond to the Fermi surfaces at six different energies for $K+(K-)$. A transformation into three separated ellipses was found when $\varepsilon_{\mathrm{F}}$ is close to the band edge. Upon introducing $D$ on a BLG, a small energy difference $\Delta \varepsilon \approx q D d \delta n / n_{\mathrm{c}}$ between $\varepsilon_{K+}$ and $\varepsilon_{K-}$ appears as illustrated in (b). It thus breaks the exact cancellation of $I_{2 \omega}$ terms for $K+$ and $K-$, giving rise to a nonzero $\sigma_{2 \omega}$. The corresponding term of $\partial I_{2 \omega} / \partial \varepsilon$ as a function of shifted energy $(\varepsilon-\Delta / 2)$ is shown in (c) for three different $\Delta$ values of 50,30, and $10 \mathrm{meV}$. In general, the magnitude of $\sigma_{2 \omega}$ is at maximum when $\varepsilon_{\mathrm{F}}$ is near the band edge, and it falls off rapidly as $\varepsilon_{\mathrm{F}}$ moves away from the Lifshitz transition energy [shaded region in (c)]. (d) The illustrations of the pseudospin states for electron-type carriers and the corresponding valley polarization in a BLG with $D \neq 0$.

at the Lifshitz transition (see Appendix C). This is an outcome from the band calculations at zero temperature, and, in principle, it can be smoothed out when taking into account a finite-temperature effect. In general, $\partial I_{2 \omega,+1} / \partial \varepsilon$ appears to be the largest when $\varepsilon_{\mathrm{F}}$ is close to the band edge with multiple separated Fermi surfaces. It then rapidly falls off as $\varepsilon_{\mathrm{F}} \geqslant \varepsilon_{\mathrm{L}}$. The decrease of $\partial I_{2 \omega,+1} / \partial \varepsilon$ with increasing $\varepsilon$ becomes faster as $D$ increases, which agrees qualitatively with the data shown in the lower panel of Fig. 3(b). We also note that the density for the maximum $R_{2 \omega}$ shown in Fig. 3(d) gives $\Delta n_{2 \mathrm{D}} \approx$ (3$8) \times 10^{10} \mathrm{~cm}^{-2}$, which is within the same order of magnitude for the density at $E_{\mathrm{L}} \approx \Delta / 2$ (see Appendix C). On the other hand, by observing Eq. (3), the sign of total $\sigma_{2 \omega}$ is dictated by $q \Delta \varepsilon \approx D \delta n$. When $\varepsilon_{\mathrm{F}}$ moves from the conduction band to the valence band with a constant $D, \delta n$ changes sign, and thus $R_{2 \omega}$ undergoes a sign reversal. On the contrary, reversing the sign for $D$ also causes a sign reversal in $\delta n$, and it thus gives no sign changes for the resulting $R_{2 \omega}$. This is also expected from the system symmetry point of view, where the longitudinal resistances of $R_{\omega}$ and $R_{2 \omega}$ are symmetric with respect to $D$ as shown in Fig. 2(c). Those behaviors are well consistent with our observations shown in Figs. 1(f) and 3(c). For an order-ofmagnitude estimation of $R_{2 \omega}$, we consider the case with $D=$ $0.6 \mathrm{~V} / \mathrm{nm}$ and a total density of $n_{2 \mathrm{D}} \approx 6 \times 10^{10} \mathrm{~cm}^{-2}$ based on our experimental data, and it gives $\Delta \varepsilon \approx 0.8 \mathrm{meV}$, which takes into account the self-consistent screening effect [21]. A rough estimation of the transport lifetime, using an effective mass of $0.024 m_{\mathrm{e}}$ [36] and a sheet resistance of $200 \mathrm{k} \Omega$, gives $\tau \approx 7$ fs. From Eq. (3) and $R_{2 \omega}=-\frac{I_{0}}{W} \frac{\sigma_{2 \omega}}{\sigma_{\omega}^{3}}$, the calculated $R_{2 \omega}$ for a bias current of $100 \mathrm{nA}$ equals $\approx 2 \mathrm{k} \Omega$ with a ratio of $R_{2 \omega} / R_{\omega} \approx 1 \%$, which is in the same order of magnitude as the measured $R_{2 \omega}$.

From $R_{2 \omega}=-\frac{I_{0}}{W} \frac{\sigma_{2 \omega}}{\sigma_{\omega}^{3}}$, the measured $R_{2 \omega}$ is expected to scale with $R_{\omega}$ with exponent larger than 3 . This is not the case, however, as shown in Fig. 4(e), giving $R_{2 \omega} \propto R_{\omega}^{\lambda}$ with $\lambda \approx$ 2.02. As it turns out, the exponent $\lambda$ is device-dependent (see Appendix D). The charge-puddle effect is known to give rise to potential fluctuations and a finite minimum conductance at the charge-neutral point in graphene-based systems [33-35]. It may enhance the conductance near the band edges, resulting in a reduced $R_{\omega}$ and spoiling the scaling of $R_{2 \omega}=R_{\omega}^{\lambda}$, particularly in the low- $D$ regime. A rough estimate from the $V_{\mathrm{bg} 0}$ value in our device gives an impurity-charge density $n_{\mathrm{imp}} \approx 1.3 \times 10^{11} \mathrm{~cm}^{-2}$, which is larger than the $\Delta n_{2 \mathrm{D}}$ shown in Fig. 3(d). This indicates the importance of the chargepuddle effects in our device, and more rigorous theoretical descriptions that include charge-puddle effects are needed. On the technical side, we also noticed a $\omega$-dependence on the measured $R_{2 \omega}$ (see Appendix E). This is attributed to the current shunting effect due to the capacitance mainly from the macroscopic gold electrodes on $\mathrm{SiO}_{2}(300 \mathrm{~nm}) / \mathrm{Si}$, which can become larger as $\varepsilon_{\mathrm{g}}$ increases in the high- $D$ regime. Such a finite capacitance in the measurement circuits can shunt the higher-frequency signals and thus cause a much reduced amplitude of $R_{2 \omega}$ as compared to $R_{\omega}$. On the other hand, we also note that the coexistence of top-gate effective and ineffective BLG regions as shown in Fig. 1(a) may lead to $\mathrm{PN}$-junction-like rectification effects. In a control device with 

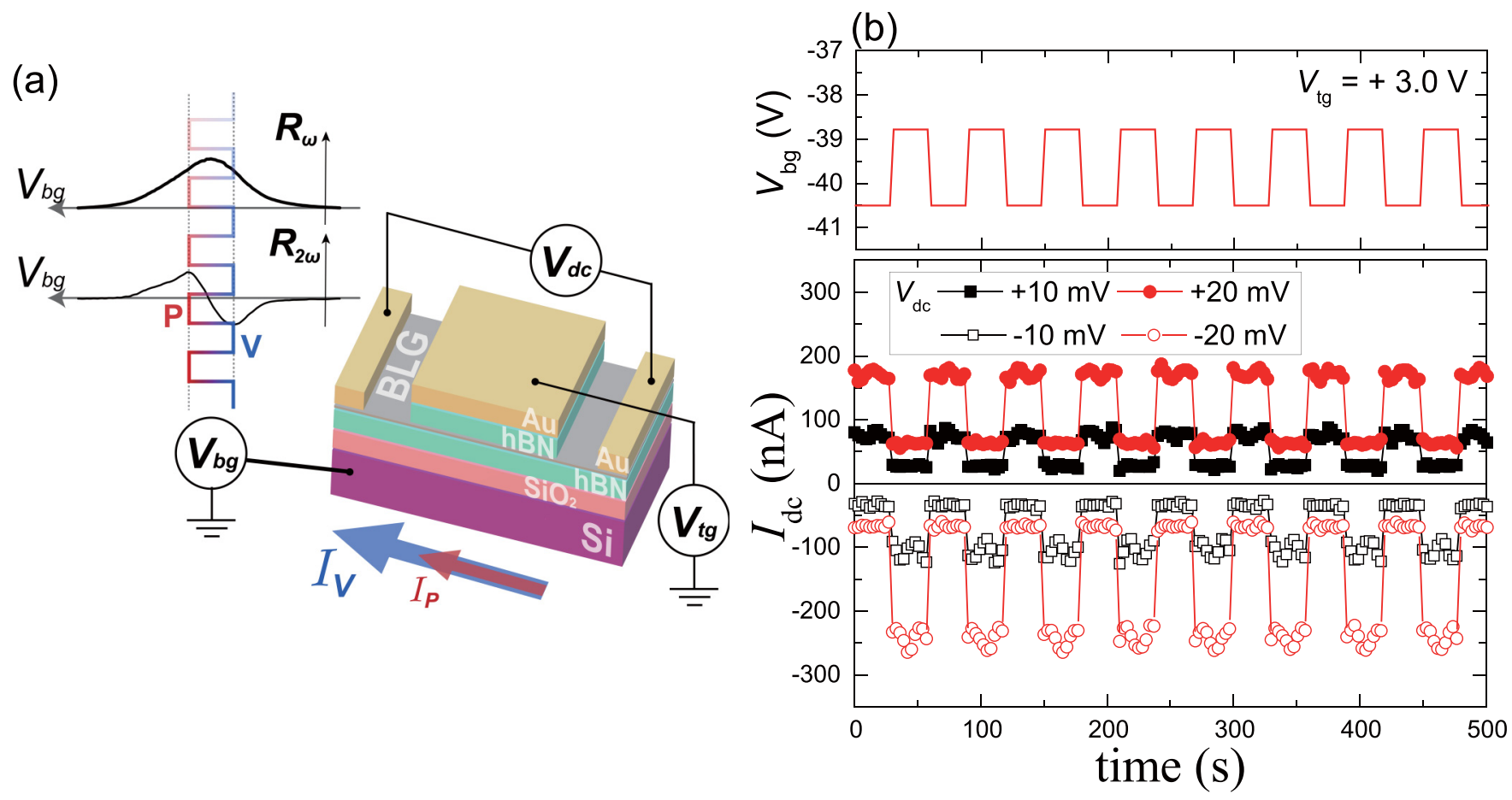

FIG. 7. A demonstration of the current rectification in a dual-gated BLG device. Part (a) illustrates the device setup and measurement procedure. A dc current $\left(I_{\mathrm{dc}}\right)$ drawn by a biased voltage $\left(V_{\mathrm{dc}}\right)$ was measured with alternating $V_{\mathrm{bg}}$ values at which the $R_{2 \omega}$ attains local extremal values. The upper panel and lower panel of (b) show the resulting time dependence of $V_{\mathrm{bg}}$ and $I_{\mathrm{dc}}$ signals, respectively, for different $V_{\mathrm{dc}}$ values.

a top-gate electrode fully covering the BLG (see Appendix D), consistent NRTE results were observed, thus excluding the rectifying contribution due to the trivial PN junction effect. Even though the issue of the sample-dependent exponent $\lambda$ requires further investigations, the observed large $R_{2 \omega} / R_{\omega}$ is well reproducible in all dual-gated BLG devices we fabricated, and, in principle, a larger $R_{2 \omega} / R_{\omega}$ can be achieved with a further increase in $D$.

As illustrated in Fig. 6(d), the directions of the eigen pseudospin states in the reduced $2 \times 2$ Hamiltonian for a BLG are determined when a bias current and thus a propagation wave vector $k$ are given, which make $\phi$ and $\pi-\phi$ angles to the current direction due to its unique chiral property. For electron-type carriers $\left(\varepsilon_{\mathrm{F}}>0\right)$, the two eigenstates of pseudospin-up $\left(S_{\mathrm{e}}^{+}\right)$and pseudospin-down $\left(S_{\mathrm{e}}^{-}\right)$correspond to electrons fully on A1 sites (upper-layer) and B2 sites (lowerlayer), respectively [29]. Therefore, the valley polarization $S$ can be defined as the charge-density difference between the two layers of BLG with a direction along the eigen pseudospin states, i.e., $S=\delta n / 2\left(S_{\mathrm{e}}^{+}-S_{\mathrm{e}}^{-}\right)$. Nevertheless, a nonzero $D$ is required to break the inversion symmetry and thus give rise to a finite value for $S \times I$. Phenomenologically, the observed NRTE in a gapped BLG can then be described by a currentdependent resistance in the form of $R=R_{0}\left[1+(-1)^{\beta} \gamma^{\prime} I\right.$. $(D \times S)$ ], where $\gamma^{\prime}$ and $S$ are the NRTE coefficient and valley polarization, respectively. $\beta$ equals 0 and 1 for electron-type and hole-type carriers, respectively, to account for the opposite signs of the $q \Delta \varepsilon$ term for the conduction band and the valence band. Upon reversing $D$, the term of $S \times I$ changes sign as required by the system symmetry, where the roles of $\mathrm{A} 1$ and B2 sites are exchanged. Therefore, there is no sign change for $R_{2 \omega}$. On the other hand, when $\varepsilon_{\mathrm{F}}$ moves to the valence band (hole-type carriers) at a constant $D, S$ attends the same sign, and the prefactor of $(-1)^{\beta}$ gives rise to the sign reversal of $R_{2 \omega}$. Finally, a scheme for a practical electric-field tuning of the current rectification effect is demonstrated in Fig. 7(a). By sourcing a dc voltage $\left(V_{\mathrm{dc}}\right)$, the measured dc current $\left(I_{\mathrm{dc}}\right)$ is found to give a high-current and low-current state, corresponding to the setting values of $V_{\mathrm{bg}}$ for extremal $R_{2 \omega \mathrm{p}}$ and $R_{2 \omega \mathrm{v}}$, respectively. As shown in Fig. 7(b), the differences in the high-current and low-current states scale linearly with the magnitude of $V_{\mathrm{dc}}$ and thus $I_{\mathrm{dc}}$. Upon reversing the sign of $V_{\mathrm{dc}}$, the high-current and low-current states are also reversed, as expected.

\section{CONCLUSION}

In conclusion, we uncovered an unusually large $R_{2 \omega}$ signal in dual-gated BLG devices, which turns out to grow with increasing bias current and also displacement field $D$. Such a NRTE in a zero magnetic field sets a clear distinction from other NRTE systems that require the breaking of the time-reversal symmetry by magnetic field. The largest ratio of $R_{2 \omega} / R_{\omega}$ for $I=100 \mathrm{nA}$ was found to be about $2.8 \%$ at $T=5 \mathrm{~K}$ and $D=+0.58 \mathrm{~V} / \mathrm{nm}$. The extremal $R_{2 \omega}$ occurs whenever the Fermi energy is shifted to the band edges, and its magnitude quickly declines when Fermi energy moves away from the Lifshitz transition energy. Those behaviors agree qualitatively well with the calculated velocity asymmetry contribution in the Boltzmann transport equation, giving a total second-harmonic conductance $\sigma_{2 \omega}=\frac{q^{3} \tau^{2}}{\pi \hbar^{2}}\left[\frac{\partial I_{2 \omega,+1}}{\partial \varepsilon}\right] \Delta \varepsilon$ with $\Delta \varepsilon \approx q D d \delta n / n_{\mathrm{c}}$. The breaking of the inversion symmetry by 
$D$ not only removes the exact cancellation between $I_{2 \omega,+1}$ and $I_{2 \omega,-1}$ but also results in valley polarization, which is crucial for the appearance of NRTE in a BLG. The scaling relation of $R_{2 \omega} \propto R_{2 \omega}^{\lambda}$ gives device-dependent values for the exponent $\lambda$ ranging from 1.3 to 4.17 , which infers a somewhat reduced increase of $R_{2 \omega}$ with $D$ in our dual-gated BLG devices. Nevertheless, the revelation of a large $R_{2 \omega}$ for the massive chiral fermions with trigonal warping enables a full electric-field tuning of the NRTE and opens up a new possibility for the valleytronics in graphene-based 2D devices.

\section{ACKNOWLEDGMENTS}

W.L.L. acknowledges the funding support from Academia Sinica (Thematic Research Program) and Ministry of Science and Technology of Taiwan (MOST Grant No. MOST 1082628-M-001-007-MY3).

\section{APPENDIX A: METHODS}

High-quality natural graphites and hexagonal boron nitrides were mechanically exfoliated by tapes and then transferred onto $300 \mathrm{~nm} \mathrm{SiO}_{2} / \mathrm{Si}$ wafers. From optical contrast analyses and atomic force microscope scannings, high-uniformity flakes with proper thicknesses were selected and sequentially stacked using the dry transfer technique with a polycarbonate/PDMS stamp. For the device shown in Fig. 1(a), the thicknesses for the top hBN-layer and bottom hBN-layer are about 22 and $6 \mathrm{~nm}$, respectively. Contact electrodes of $\operatorname{Ti}(5 \mathrm{~nm}) / \mathrm{Au}(30 \mathrm{~nm})$ were then fabricated by an electron beam lithography facility. The device measurements were carried out in a closed-cycle cryostat that provides stable sample temperature above $T=5 \mathrm{~K}$. A lock-in amplifier was used for the measurements of $R_{\omega}$ and $R_{2 \omega}$ simultaneously with a bias current at a low frequency of $18.111 \mathrm{~Hz}$.

\section{APPENDIX B: THE SHEET DENSITY AND HALL MOBILITY}

To characterize the properties of the bilayer graphene sample, we have performed Hall measurements at $T=5 \mathrm{~K}$ with $V_{\mathrm{tg}}=0$. The Hall resistance was found to be field-linear in weak fields up to $0.5 \mathrm{~T}$, and the corresponding sheet density $n_{2 \mathrm{D}}$ was obtained from the Hall coefficient $R_{H}=\frac{1}{n_{2 \mathrm{D}} e}$, where $e$ is the elementary charge. We measured the Hall resistance at 0.5 and $-0.5 \mathrm{~T}$ and estimated the carrier density with respect to $V_{\mathrm{bg}}$, as shown in Fig. 8(a). We have also tried to evaluate the capacitance of the bottom-gate in our dual-gated device. The capacitance $C=\frac{\Delta n_{2 \mathrm{D}} e}{\Delta V_{\mathrm{bg}}}$ can be determined from the slope for the curve of carrier density as a function of voltage of the bottom-gate. The capacitance values in the electron-doping and hole-doping regions are around 91.34 and $109.72 \mathrm{aF} / \mu \mathrm{m}^{2}$, respectively, which are well consistent with the nominal value of around $110 \mathrm{aF} / \mu \mathrm{m}^{2}$ estimated from a simple parallel plate capacitor model. The corresponding mobility $\mu$ as a function of the bottom-gate is shown in Fig. 8(b), using the relation of conductivity $\sigma=n_{2 \mathrm{D}} e \mu$. The mobility is more than $10000 \mathrm{~cm}^{2} / \mathrm{V} \mathrm{s}$ in the electron-doping region, and it is slightly higher for the hole-doping region. We do note some anomaly in the hole-doping region near $n_{2 \mathrm{D}}=(0.5-$ 1.0) $\times 10^{12} \mathrm{~cm}^{-2}$, which is likely due to the contribution from top-gate ineffective regions between the voltage leads.

\section{APPENDIX C: CALCULATIONS OF $\sigma_{2 \omega}$ BASED ON THE BOLTZMANN TRANSPORT EQUATION}

The equilibrium Fermi-Dirac distribution function is given by $f_{0}=\frac{1}{e^{(\epsilon-\mu) / k_{B} T}+1}$. The total electron distribution is $f_{\overrightarrow{\mathrm{k}}}=f_{0}+g_{\overrightarrow{\mathrm{k}}}$, where $g_{\overrightarrow{\mathrm{k}}}$ refers to the nonequilibrium distribution function. Using a relaxation approximation with $\left(\frac{\partial f_{\overrightarrow{\mathrm{k}}}}{\partial t}\right)_{\text {collision }}=-\frac{g_{\overrightarrow{\mathrm{k}}}}{\tau}$, we set $\vec{E}=E_{\mathrm{x}} \hat{i}$ and keep terms up to $E^{2}$
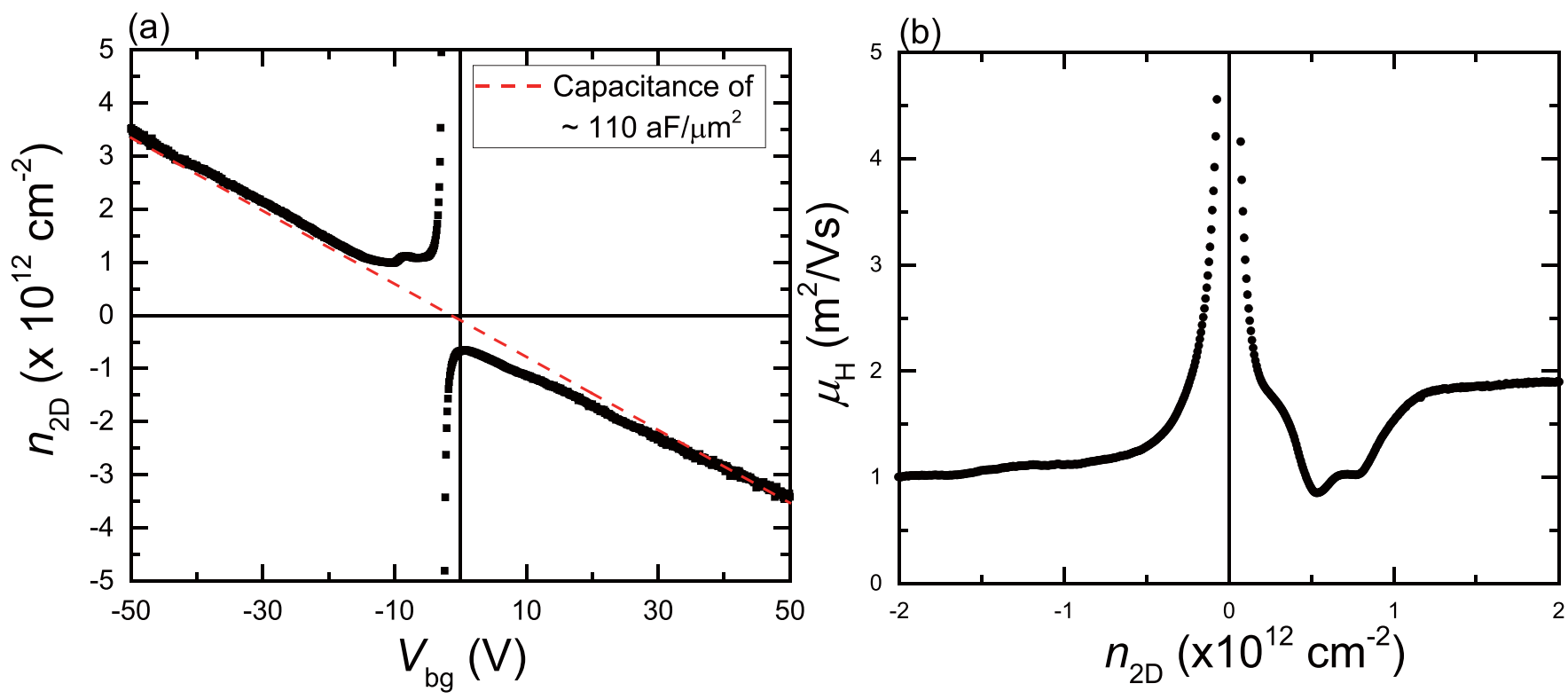

FIG. 8. Hall effect measurements. (a) The sheet density $n_{2 \mathrm{D}}$ as a function of $V_{\mathrm{bg}}$. The red dashed line is a curve with a capacitance of $\approx 110$ $\mathrm{aF} \mu \mathrm{m}^{-2}$ via the bottom gate. (b) The extracted mobility $\mu$ as a function of $V_{\mathrm{bg}}$. 

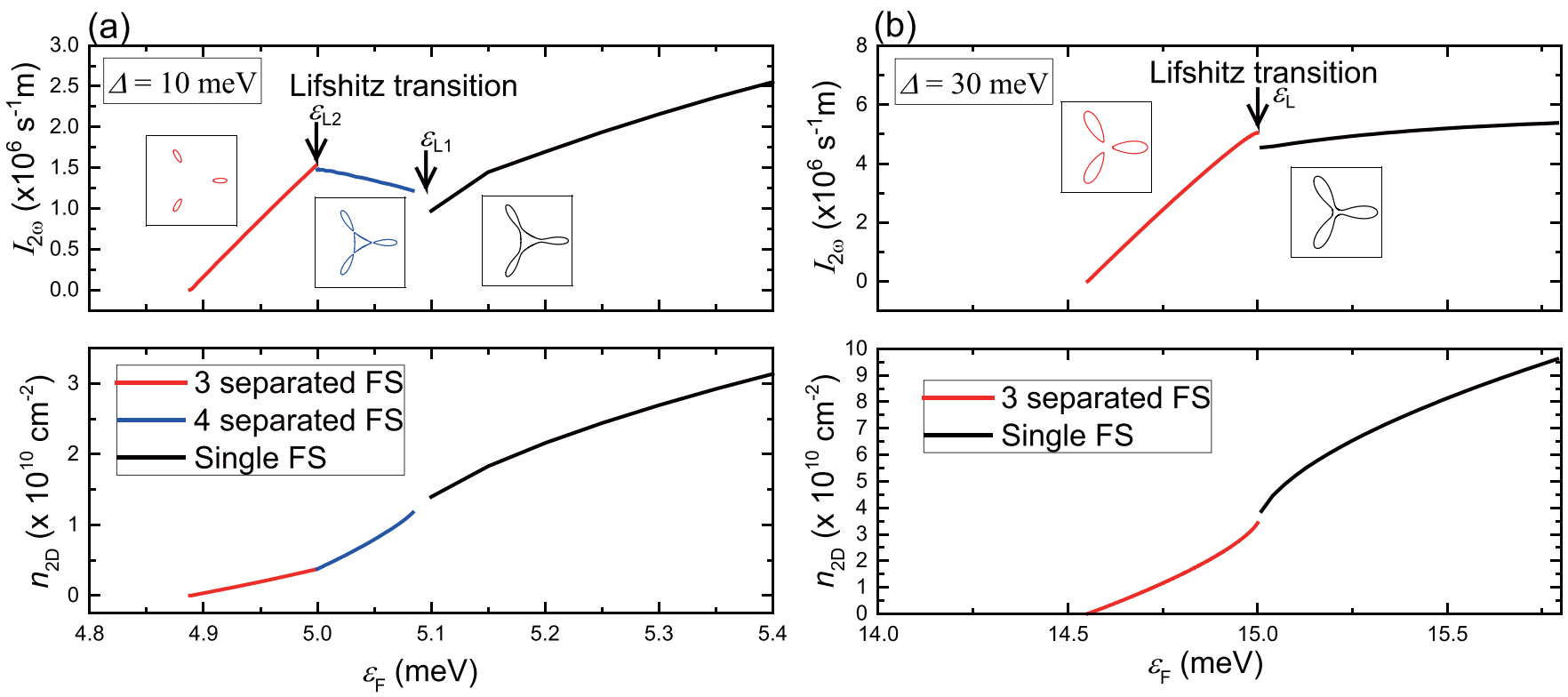

FIG. 9. The numerically calculated $I_{2 \omega}$ and $n_{2 \mathrm{D}}$ for the $K+$ valley at different values of asymmetry parameter $\Delta$. The calculated $I_{2 \omega}$ and $n_{2 \mathrm{D}}$ as a function of the Fermi energy $\varepsilon_{\mathrm{F}}$ are plotted in the upper panel and lower panel of (a) for $\Delta=10$ meV, respectively. Part (c) shows the calculated $I_{2 \omega}$ and $n_{2 \mathrm{D}}$ for $\Delta=30 \mathrm{meV}$.

for $g_{\overrightarrow{\mathrm{k}}}$, and then

$g_{\overrightarrow{\mathrm{k}}}=-\frac{q \vec{E} \tau}{\hbar} \frac{\partial\left(f_{0}+g_{\overrightarrow{\mathrm{k}}}\right)}{\vec{k}}, \quad \approx-\frac{q E_{\mathrm{x}} \tau}{\hbar} \frac{\partial f_{0}}{\partial k_{\mathrm{x}}}+\left(\frac{q E_{\mathrm{x}} \tau}{\hbar}\right)^{2} \frac{\partial^{2} f_{0}}{\partial k_{\mathrm{x}}^{2}}$

The current density for a two-dimensional system is then

$$
\begin{aligned}
j_{\mathrm{x}} & =2 q \int v_{\overrightarrow{\mathrm{k}}} g_{\overrightarrow{\mathrm{k}}} \frac{d^{2} \vec{k}}{(2 \pi)^{2}} \\
& =\left[-\frac{q^{2}}{\hbar} \int \frac{d^{2} \vec{k}}{2 \pi^{2}} \tau v_{\overrightarrow{\mathrm{k}}} \frac{\partial f_{0}}{\partial k_{\mathrm{x}}}\right] E_{\mathrm{x}}+\left[\frac{q^{3}}{\hbar^{2}} \int \frac{d^{2} \vec{k}}{2 \pi^{2}} \tau^{2} v_{\overrightarrow{\mathrm{k}}} \frac{\partial^{2} f_{0}}{\partial k_{\mathrm{x}}^{2}}\right] E_{\mathrm{x}}^{2} \\
& \equiv \sigma_{\omega} E_{\mathrm{x}}+\sigma_{2 \omega} E_{\mathrm{x}}^{2} .
\end{aligned}
$$

Therefore, the second-harmonic conductance can be expressed as [23]

$$
\begin{aligned}
\sigma_{2 \omega} & =\frac{q^{3}}{\hbar^{2}} \int \frac{d^{2} \vec{k}}{2 \pi^{2}} \tau^{2} v_{\overrightarrow{\mathrm{k}}} \frac{\partial^{2} f_{0}}{\partial k_{\mathrm{x}}^{2}} \\
& =\frac{q^{3} \tau^{2}}{2 \pi \hbar} \oint d S_{\mathrm{F}} \cos ^{3} \phi\left[3 \frac{\left|v_{\mathrm{k}}\right|}{v_{\mathrm{k}}} \frac{\partial v_{\mathrm{k}}}{\partial p}-\frac{\partial\left|v_{\mathrm{k}}\right|}{\partial p}\right]_{\varepsilon_{\mathrm{F}}} \\
& \equiv \frac{q^{3} \tau^{2}}{2 \pi \hbar^{2}} I_{2 \omega},
\end{aligned}
$$

where $d S_{\mathrm{F}}=p d \phi / \hbar$ at the Fermi surface, $v_{\mathrm{k}}=\partial \varepsilon / \partial p$, and $I_{2 \omega} \equiv \oint d \phi p \cos ^{3} \phi\left[3 \frac{\left|v_{\mathrm{k}}\right|}{v_{\mathrm{k}}} \frac{\partial v_{\mathrm{k}}}{\partial p}-\frac{\partial\left|v_{\mathrm{k}}\right|}{\partial p}\right]_{\varepsilon_{\mathrm{F}}}$ is the path integral along closed Fermi surfaces. Here, we assume that $\tau$ is independent of $p$ and $\phi$.

By numerically calculating $v_{\mathrm{k}}$ and $\partial v_{\mathrm{k}} / \partial p$ for a gapped BLG with trigonal warping, $I_{2 \omega}$ and thus $\sigma_{2 \omega}$ can be obtained. Using the energy dispersion for a gapped BLG with trigonal warping [Eq. (1)], the $I_{2 \omega}$ term can be numerically calculated and shown in the upper panels of Figs. 9(a) and 9(b) for two different asymmetry parameters of $\Delta=10$ and $30 \mathrm{meV}$, respectively, where the corresponding calculated sheet densities $n_{2 \mathrm{D}}$ are shown in the lower panel. The inset cartoons show the corresponding Fermi surfaces in different $\varepsilon_{\mathrm{F}}$ regimes. For $\Delta=10 \mathrm{meV}$ [Fig. 9(a)], there are two Lifshitz transitions at energies of $\varepsilon_{\mathrm{L} 1}$ and $\varepsilon_{\mathrm{L} 2} \cdot \varepsilon_{\mathrm{L} 1}$ equals $\approx 5.09 \mathrm{meV}$, corresponding to a transition from a single Fermi surface (black line) to four separated Fermi surfaces (blue line). As $\varepsilon_{\mathrm{F}}$ keeps decreasing, another transition into three separated Fermi surfaces (red line) occurs at $\varepsilon_{\mathrm{L} 2} \approx 5.00 \mathrm{meV}$. Figure 9 (b) shows the results for $\Delta=30 \mathrm{meV}$, where only one Lifshitz transition from a single Fermi surface to three separated Fermi surfaces was observed at $\varepsilon_{\mathrm{L}} \approx 15 \mathrm{meV}$. We note that both calculated $I_{2 \omega}$ and $n_{2 \mathrm{D}}$ values show abrupt changes at Lifshitz transition energies. The sheet density at $\varepsilon_{\mathrm{L} 1}\left(\varepsilon_{\mathrm{L}}\right)$ for $\Delta=10 \mathrm{meV}(30 \mathrm{meV})$ equals $\approx 1.3(3.9) \times 10^{10} \mathrm{~cm}^{-2}$, which turns out to increase gradually with $\Delta$. Within our experimental $D$ values, the sheet density at the Lifshitz transition is expected to be in the range of $\approx(1-10) \times 10^{10} \mathrm{~cm}^{-2}$, which is in the same order of magnitude as the sheet density that we observed in experiment for the occurrence of a maximum $\left|R_{2 \omega}\right|$ [Fig. 3(d)].

The measured quantity of $R_{2 \omega}$ can be related to $\sigma_{\omega}$ and $\sigma_{2 \omega}$ by inverting the current density equation shown in Eq. (C2) [14], giving

$$
E_{\mathrm{x}} \approx\left[1 / \sigma_{\omega}\right] j_{\mathrm{x}}-\left[\sigma_{2 \omega} / \sigma_{\omega}^{3}\right] j_{\mathrm{x}}^{2} .
$$

Therefore, the magnitude of the measured second-harmonic sheet resistance $R_{2 \omega}$ can be shown to be

$$
R_{2 \omega} \equiv \frac{V_{2 \omega}}{I_{0}} \frac{W}{L}=-\frac{I_{0}}{W} \frac{\sigma_{2 \omega}}{\sigma_{\omega}^{3}},
$$

where $V_{2 \omega}$ is the measured second-harmonic voltage, $I_{0}$ is the magnitude of the driving current, and $W(L)$ is the device width (length between voltage electrodes). 
(a)

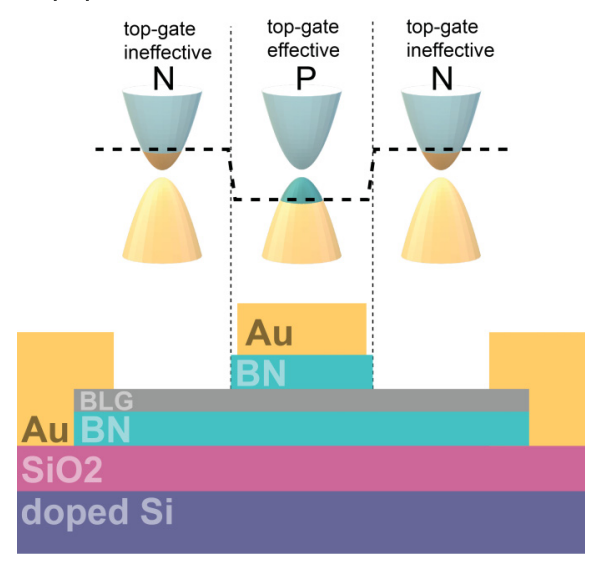

(b)

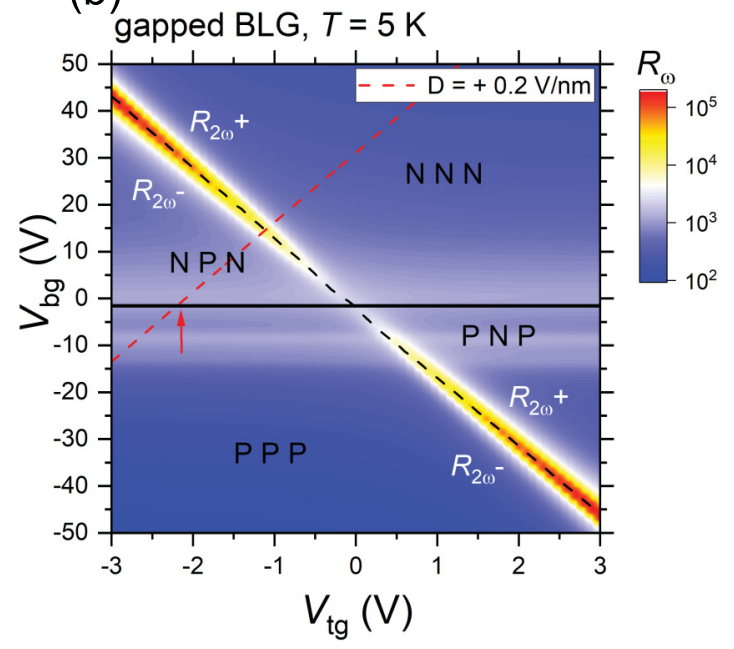

FIG. 10. Semiconducting PN phase diagram of a dual-gated BLG device. (a) An illustration of a dual-gated BLG device with top-gate effective and ineffective regions. (b) Sheet resistance of BLG as a function of bottom gate and top gate at $5 \mathrm{~K}$. The dashed black line is a visual guide showing the charge neutrality of the top-gate effective region. The dashed and solid black lines divide the phase diagram into four parts of different polarities. The observed sign of $R_{2 \omega}$ is also marked in (b). The red dashed line indicates the $D$-constant line of $D=+0.2 \mathrm{~V} / \mathrm{nm}$ across three different PN phases.

\section{APPENDIX D: DISCUSSIONS OF THE POSSIBLE PN JUNCTION EFFECT AND $\boldsymbol{R}_{2 \omega}$ IN OTHER DUAL-GATED BLG DEVICES}

Electron/hole-doping concentration in BLG is manipulated by a top-gate and a bottom-gate. The silicon wafer is used as a bottom-gate, so the whole area of BLG is affected by the bottom-gate. However, the area of the top-gate is limited and only a part of BLG can be affected by the top-gate, as shown in Fig. 1(a). Thus, the BLG device is divided into three regions, as illustrated in Fig. 10(a), where only the middle region is top-gate effective and the other two regions are only bottom-gate effective (or top-gate ineffective). The sheet resistance $\left(R_{\omega}\right)$ as a function of $V_{\mathrm{bg}}$ and $V_{\mathrm{tg}}$ at $5 \mathrm{~K}$ is shown in Fig. 10(b). The dashed line shows the locations for $R_{\omega}$ peaks and corresponds to the charge neutrality for the top-gate effective region of a gapped BLG. The solid line represents $V_{\mathrm{bg}}=$ $V_{\mathrm{bg} 0}(\approx-1.86 \mathrm{~V})$, which gives a maximum $R_{\omega}$ at zero $V_{\mathrm{tg}}$, which separates the P-type and $\mathrm{N}$-type phases for the top-gate ineffective regions. These two lines divide the resistance map into four phases, representing four different combinations of NNN, NPN, PPP, and PNP based on the Fermi level location as illustrated in Fig. 10(a). It is known that a PN junction results in a current rectification effect. However, our dualgated device comprises two PN junctions that work more like a transition with gate-tunable conductance across the device, and it is not likely to exhibit current-directional-dependent resistance for a single PN junction. Starting from NPN phase, for example, the conductance reaches a minimum near the dashed-line region when moving along the $D$-constant line to the NNN phase as expected for a transistor-like behavior. Supposedly, there is somehow a difference in the two topgate ineffective regions [Fig. 10(a)], and it thus may cause certain current-directional-dependent effects. It still cannot explain the observed complex $R_{2 \omega}$ behaviors as described in the main text, including the sign changes and also the rapid decays of its magnitude away from the band edges. On the other hand, when moving along the $D=+0.2 \mathrm{~V} / \mathrm{nm}$ line [red dashed line in Fig. 10(b)], we shall observe another large $R_{2 \omega}$ when moving from NPN to PPP phases, but this was not happening. As also shown in Fig. 11(a), a control device with the top-gate electrode fully covering the voltage leads was fabricated to ensure no PN junctions within the voltage leads, and a consistent NRTE effect was observed. We argue, therefore, that the observed large $R_{2 \omega}$ is not likely due to the trivial PN semiconducting junction or diode-related effects.

To avoid a possible PN-junction-like effect, we fabricated a dual-gated BLG device with the top-gate electrode fully covering the voltage leads as shown in Fig. 11(a). The Hall mobility is about $5000 \mathrm{~cm}^{2} / \mathrm{V} \mathrm{s}$, which is slightly lower than the device shown in Fig. 1(a) due to the additional Hall-bar patterning process on BLG. The resulting $R_{2 \omega}$ data are shown in Fig. 11(b), where $R_{2 \omega}$ attends a peak near the conduction-band edge and then rapidly drops to a valley near the valence-band edge when moving along the $D$-constant contour line. The corresponding scaling plot of $R_{2 \omega}$ versus $R_{\omega}$ in a logarithmic scale is shown in Fig. 11(c), giving a relation of $R_{2 \omega} \propto R_{\omega}^{4.17}$ and a maximum ratio of $R_{2 \omega} / R_{\omega} \approx 1.5 \%$. Another dual-gated BLG device was fabricated with a much narrow top-gate electrode of only about $0.8 \mu \mathrm{m}$ as shown in Fig. 11(d). The Hall mobility is about $10^{5} \mathrm{~cm}^{2} / \mathrm{V} \mathrm{s}$ at $n_{2 \mathrm{D}} \approx$ $1 \times 10^{11} \mathrm{~cm}^{-2}$. The measured $R_{2 \omega}$ are shown in Fig. 11(e) with an exponent $\lambda \approx 1.87$ [Fig. 11(f)]. The maximum ratio of $R_{2 \omega} / R_{\omega}$ is about $7 \%$. For more than five dual-gated BLG devices with Hall mobilities ranging from 3000 to $10^{5} \mathrm{~cm}^{2} / \mathrm{V} \mathrm{s}$, $R_{2 \omega}$ signals appear in all the devices and also show consistent behavior and a similar order of magnitude for NRTE as described in the main text. However, the exponent $\lambda$ is shown 
(a)

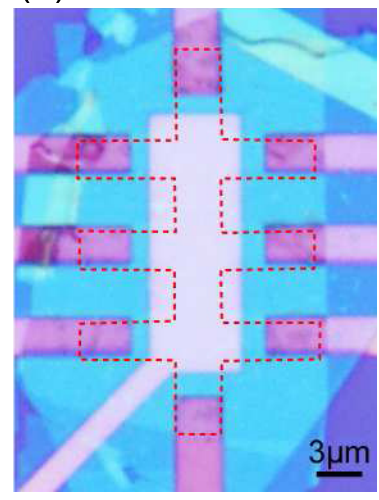

(d)

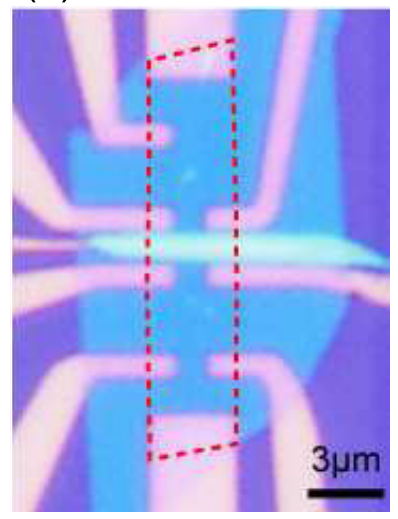

(b)

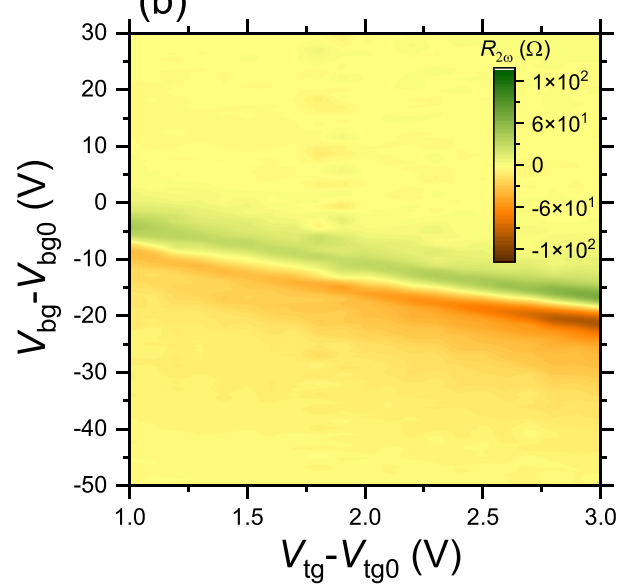

(e)

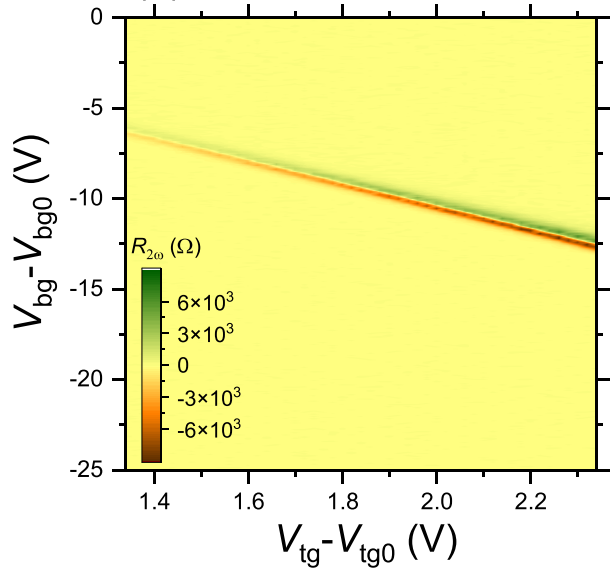

(c)

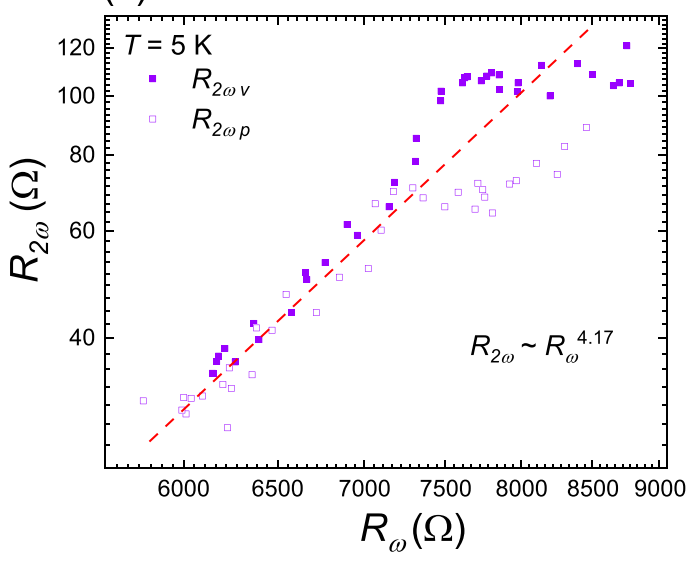

(f)

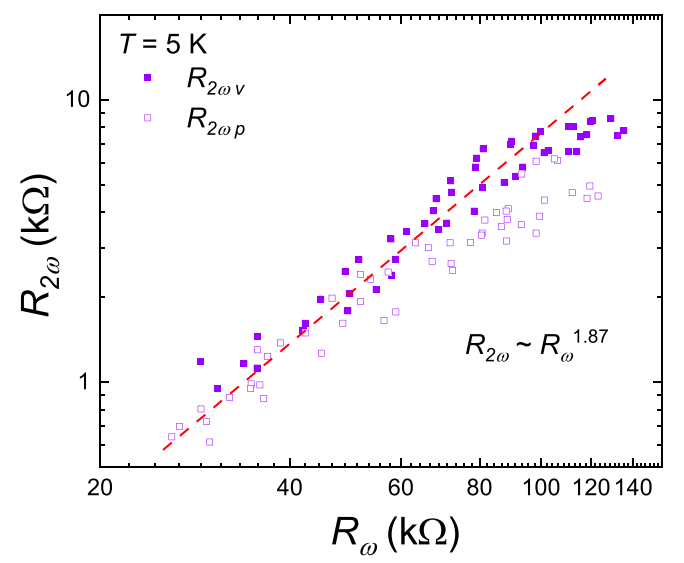

FIG. 11. The measured $R_{2 \omega}$ for other dual-gated BLG devices. (a) The optical microscope image of a dual-gated BLG with a top gate fully covering the voltage leads. The corresponding $R_{2 \omega}$ as a function of $V_{\mathrm{bg}}$ and $V_{\mathrm{tg}}$ at $T=5 \mathrm{~K}$ is shown in (b), which shows a consistent behavior with that described in the main text. (c) The scaling plot of $R_{2 \omega}$ vs $R_{\omega}$ gives an exponent $\lambda \approx 4.17$. On the other hand, the results for another dual-gated BLG device with a much narrower top-gate electrode is shown in (d), (e), and (f), giving an exponent $\lambda \approx 1.87$.

to be sample-dependent with values ranging from about 1.3 to 4.17.

\section{APPENDIX E: THE FREQUENCY DEPENDENCE AND COMPARISON TO RESULTS FROM PULSED DC MEASUREMENTS}

For ac measurements, a change in the driving frequency turns out to cause a variation in both measured $R_{\omega}$ and $R_{2 \omega}$. The frequency dependences of $R_{\omega}$ and $R_{2 \omega}$ are shown in Figs. 12(a) and 12(b), respectively. Both $R_{\omega}$ and $R_{2 \omega}$ drop with increasing driving frequency, where the decay in $R_{2 \omega}$ with increasing frequency appears to be more significant. This indicates the capacitance effect in our measurements circuits, which is mainly derived from the macroscopic gold electrodes. The whole dual-gated device comprises a template with macroscopic gold pads with size up to about $3 \mathrm{~mm}$. An estimated capacitance of $\mathrm{Au} / \mathrm{SiO}_{2}(300 \mathrm{~nm}) / \mathrm{Si}$ can be as large as $150 \mathrm{pF}$, and it gives an impedance of about $1 \mathrm{M} \Omega$ at $1 \mathrm{kHz}$, which is comparable to the $R_{\omega 0}$ at low temperature. Therefore, the ac measurements for probing the NRTE in dual-gated devices should be carried out in the low-frequency regime to avoid the shunting of the current via the capacitive coupling to electrodes. We note that this is particularly important for the measurements near the band edges in the high- $D$ regime, where the sheet resistance of a gapped BLG rises up rapidly. For a low driving frequency of about $18 \mathrm{~Hz}$, the imaginary component of $R_{2 \omega}$ turns out to dominate when setting with the same phase value as that for the $R_{\omega}$ detection, which thus confirms a phase difference of about $\pi / 2$ between the $R_{\omega}$ and $R_{2 \omega}$ signals as expected for NRTE. We would also like to remark that $\mathrm{Ti} / \mathrm{Au}$ are commonly used as contact electrodes for graphene-based devices for low contact resistance. In addition, the BLG regions that were in contact with $\mathrm{Ti} / \mathrm{Au}$ are only coupled to the bottom gate $\left(V_{\mathrm{bg}}\right)$ and should be consider as heavily doped regions when the dual-gated device was set at a large $D$ condition. Therefore, the Schottky barriers are not likely to form and give rise to trivial current rectification effects in our device.

We have also performed pulsed dc measurement to confirm the nonreciprocal transport effect. One of the concerns of dc pulse measurement is the onset of excitation. When the current excitation is suddenly lifted from zero to a set point, there is a finite rise time for the responding voltage. 
(a)

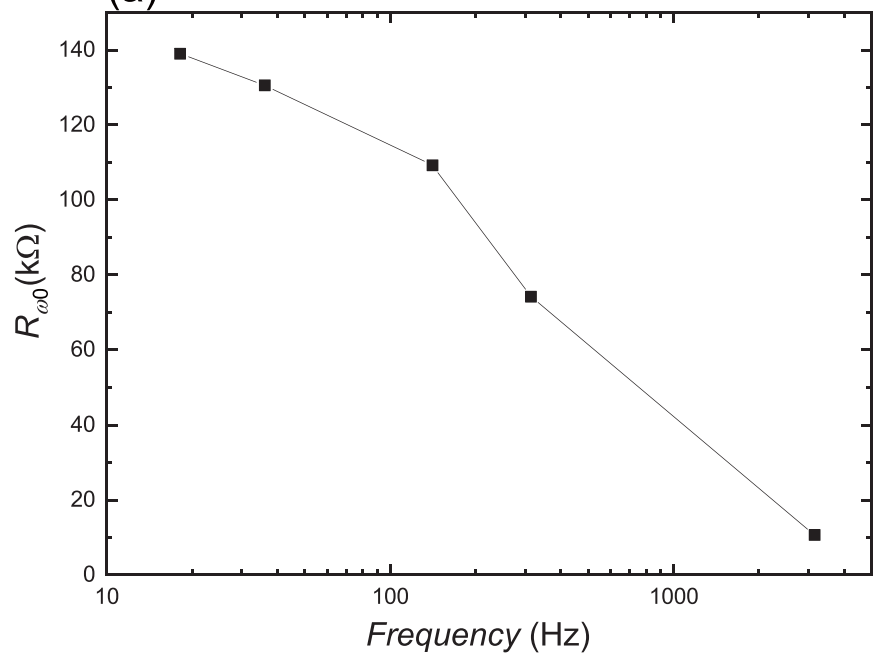

(b)

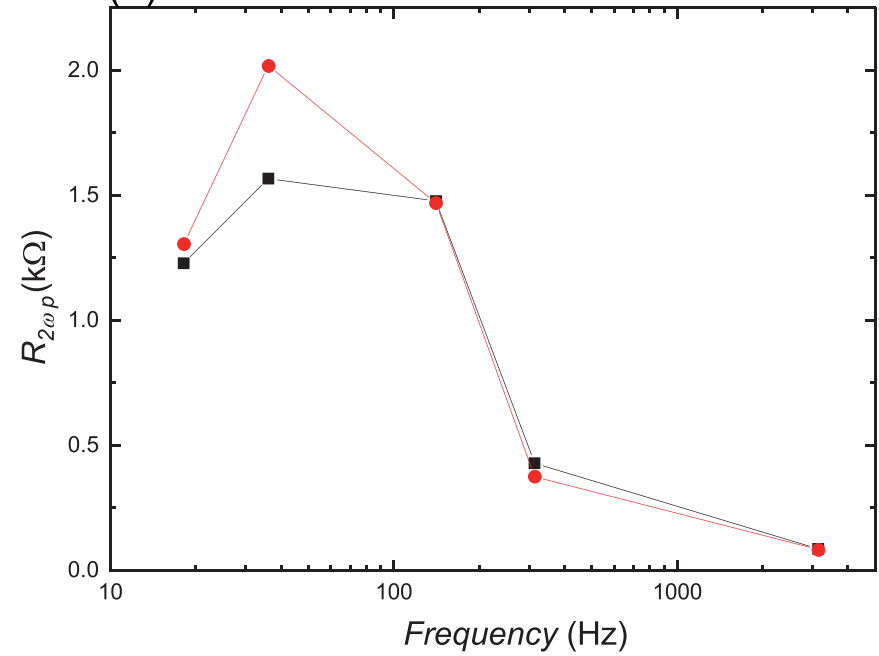

FIG. 12. Frequency dependence of the measured signals. Parts (a) and (b) are the first-harmonic sheet resistance and the second-harmonic resistance, respectively, as a function of ac driving frequency in logarithmic scale.

To minimize the Joule heating effect, the proper width for the current pulse and also a suitable delay time are carefully chosen to ensure a reliable measurement. For consistency, at each $V_{\mathrm{bg}}$ set point, the resistance data are derived from statistical average data of 50 pulsed dc measurements, where each measurement comprises a current pulse of $10 \mathrm{~ms}$ width and a voltage measurement with $8 \mathrm{~ms}$ source delay. The measured resistance as a function of the bottom-gate through dc pulse mode measurement using 20 and $-20 \mathrm{nA}$ under $V_{\mathrm{tg}}=$ $-2.5 \mathrm{~V}$ is shown in Fig. 13(a), which demonstrates an observable difference in the resistance near the local maximum of $R_{\omega}$. Thus, the current-direction-dependent resistance is again (a)

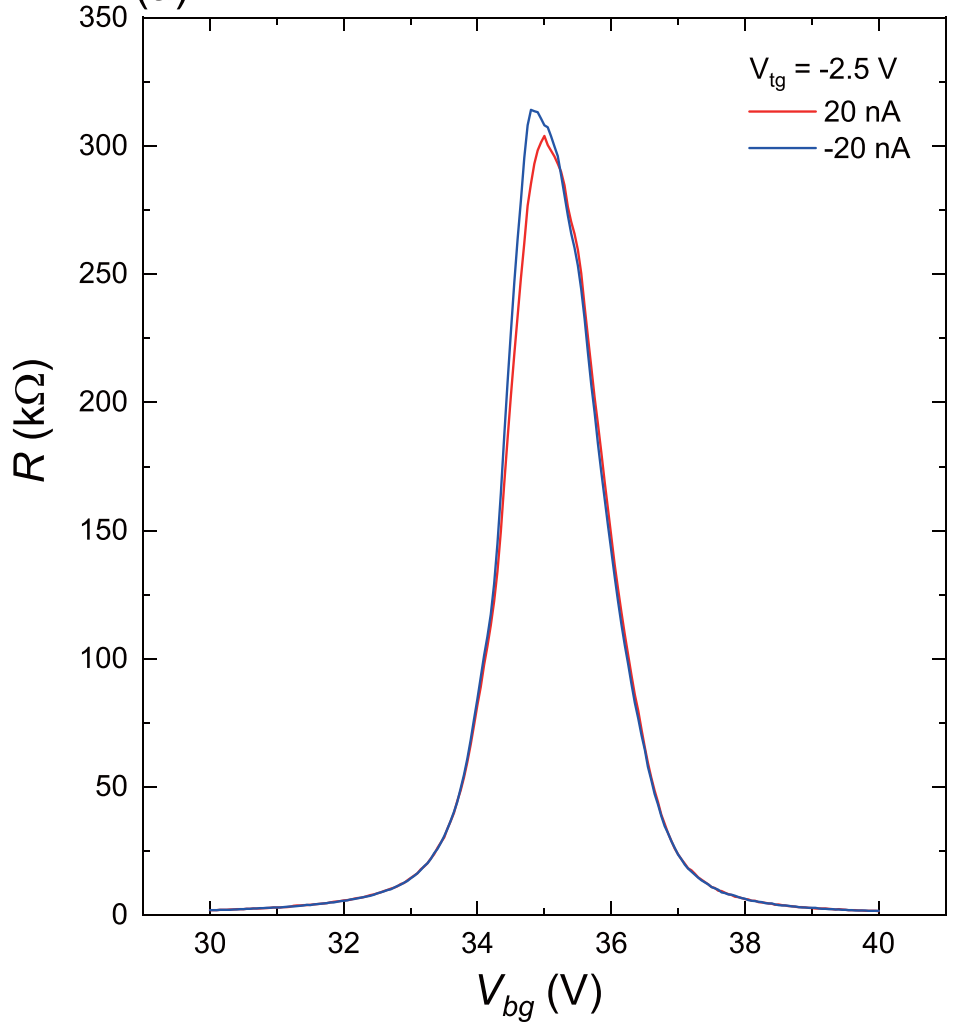

(b)
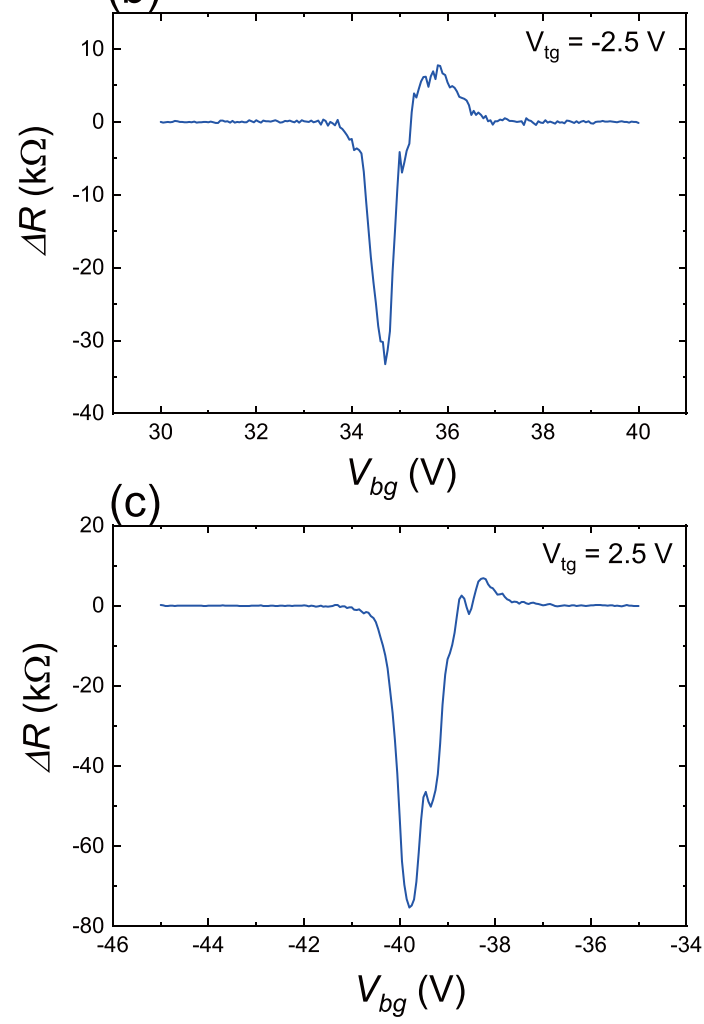

FIG. 13. Pulsed dc measurements. (a) Sheet resistance as a function of bottom gate with the top gate fixed at $-2.5 \mathrm{~V}$ using opposite current directions by a dc pulse measurement. Parts (b) and (c) show the difference of sheet resistances as a function of bottom-gate with top-gate values fixed at -2.5 and $2.5 \mathrm{~V}$, respectively. 
verified under pulsed dc measurement. The resistance change $\Delta R=R_{20 \mathrm{nA}}-R_{-20 \mathrm{nA}}$ as a function of the bottom-gate with $V_{\mathrm{tg}}=-2.5$ and $2.5 \mathrm{~V}$ is plotted in Figs. 13(b) and 13(c), respectively. A noticeable $\Delta R$ happens near $V_{\mathrm{bg}}=35 \mathrm{~V}$ with $V_{\mathrm{tg}}=-2.5 \mathrm{~V}$ and $V_{\mathrm{bg}}=-39 \mathrm{~V}$ with $V_{\mathrm{tg}}=2.5 \mathrm{~V}$. $\Delta R$ changes sign when the type of carrier changes. The observed $\Delta R$ first attains a peak and then drops rapidly to reach a valley as gate voltage decreases. Those results are consistent with the behavior in ac measurements shown in the main text.
[1] G. L. J. A. Rikken and P. Wyder, Magnetoelectric Anisotropy in Diffusive Transport, Phys. Rev. Lett. 94, 016601 (2005).

[2] Y. Tokura and N. Nagaosa, Nonreciprocal responses from noncentrosymmetric quantum materials, Nat. Commun. 9, 3740 (2018).

[3] Y. Ogawa, Y. Kaneko, J. P. He, X. Z. Yu, T. Arima, and Y. Tokura, Magnetization-Induced Second Harmonic Generation in a Polar Ferromagnet, Phys. Rev. Lett. 92, 047401 (2004).

[4] I. Grinberg, D. V. West, M. Torres, G. Gou, D. M. Stein, L. Wu, G. Chen, E. M. Gallo, A. R. Akbashev, P. K. Davies et al., Perovskite oxides for visible-light-absorbing ferroelectric and photovoltaic materials, Nature (London) 503, 509 (2013).

[5] F. Pop, P. Auban-Senzier, E. Canadell, G. L. J. A. Rikken, and N. Avarvari, Electrical magnetochiral anisotropy in a bulk chiral molecular conductor, Nat. Commun. 5, 3757 (2014).

[6] T. Ideue, K. Hamamoto, S. Koshikawa, M. Ezawa, S. Shimizu, Y. Kaneko, Y. Tokura, N. Nagaosa, and Y. Iwasa, Bulk rectification effect in a polar semiconductor, Nat. Phys. 13, 578 (2017).

[7] T. Yokouchi, N. Kanazawa, A. Kikkawa, D. Morikawa, K. Shibata, T. Arima, Y. Taguchi, F. Kagawa, and Y. Tokura, Electrical magnetochiral effect induced by chiral spin fluctuations, Nat. Commun. 8, 866 (2017).

[8] R. Wakatsuki, Y. Saito, S. Hoshino, Y. M. Itahashi, T. Ideue, M. Ezawa, Y. Iwasa, and N. Nagaosa, Nonreciprocal charge transport in noncentrosymmetric superconductors, Sci. Adv. 3, e1602390 (2017).

[9] K. Yasuda, H. Yasuda, T. Liang, R. Yoshimi, A. Tsukazaki, K. S. Takahashi, N. Nagaosa, M. Kawasaki, and Y. Tokura, Nonreciprocal charge transport at topological insulator/superconductor interface, Nat. Commun. 10, 2734 (2019).

[10] D. Choe, M. J. Jin, S. I. Kim, H. J. Choi, J. Jo, I. Oh, J. Park, H. Jin, H. C. Koo, B. C. Min et al., Gate-tunable giant nonreciprocal charge transport in noncentrosymmetric oxide interfaces, Nat. Commun. 10, 4510 (2019).

[11] F. Ando, Y. Miyasaka, T. Li, J. Ishizuka, T. Arakawa, Y. Shiota, T. Moriyama, Y. Yanase, and T. Ono, Observation of superconducting diode effect, Nature (London) 584, 373 (2020).

[12] Y. M. Itahashi, T. Ideue, Y. Saito, S. Shimizu, T. Ouchi, T. Nojima, and Y. Iwasa, Nonreciprocal transport in gate-induced polar superconductor $\mathrm{SrTiO}_{3}$, Sci. Adv. 6, eaay9120 (2020).

[13] C. O. Avci, A. Garello, A. Ghosh, M. Gabureac, S. F. Alvarado, and P. Gambardella, Unidirectional spin Hall magnetoresistance in ferromagnet/normal metal bilayers, Nat. Phys. 11, 570 (2015).

[14] K. Yasuda, A. Tsukazaki, R. Yoshimi, K. S. Takahashi, M. Kawasaki, and Y. Tokura, Large Unidirectional Magnetoresistance in a Magnetic Topological Insulator, Phys. Rev. Lett. 117, 127202 (2016).

[15] Y. Lv, J. Kally, D. Zhang, J. S. Lee, M. Jamali, N. Samarth, and J. P. Wang, Unidirectional spin-Hall and Rashba-Edelstein magnetoresistance in topological insulator-ferromagnet layer heterostructures, Nat Commun. 9, 111 (2018).

[16] K. Kechedzhi, V. I. Fal'ko, E. McCann, and B. L. Altshuler, Influence of Trigonal Warping on Interference Effects in Bilayer Graphene, Phys. Rev. Lett. 98, 176806 (2007).

[17] L. Fu, Hexagonal Warping Effects in the Surface States of the Topological Insulator $\mathrm{Bi}_{2} \mathrm{Te}_{3}$, Phys. Rev. Lett. 103, 266801 (2009).

[18] P. He, S. S. L. Zhang, D. P. Zhu, Y. Liu, Y. Wang, J. W. Yu, G. Vignale, and $\mathrm{H}$. Yang, Bilinear magnetoelectric resistance as a probe of three-dimensional spin texture in topological surface states, Nat. Phys. 14, 495 (2018)

[19] E. V. Castro, K. S. Novoselov, S. V. Morozov, N. M. R. Peres, J. M. B. Lopes dos Santos, J. Nilsson, F. Guinea, A. K. Geim, and A. H. Castro Neto, Biased Bilayer Graphene: Semiconductor with a Gap Tunable by the Electric Field Effect, Phys. Rev. Lett. 99, 216802 (2007)

[20] C. R. Wang, W. S. Lu, L. Hao, W. L. Lee, T. K. Lee, F. Lin, I. C. Cheng, and J. Z. Chen, Enhanced Thermoelectric Power in Dual-Gated Bilayer Graphene, Phys. Rev. Lett. 107, 186602 (2011).

[21] E. McCann and M. Koshino, The electronic properties of bilayer graphene, Rep. Prog. Phys. 76, 056503 (2013).

[22] E. McCann and V. I. Fal'ko, Landau-Level Degeneracy and Quantum Hall Effect in a Graphite Bilayer, Phys. Rev. Lett. 96 , 086805 (2006).

[23] J. M. Ziman, Principle of the Theory of Solids (Cambridge University Press, Cambridge, 1972), Chap. 7.

[24] A. S. Mayorov, D. C. Elias, M. Mucha-Kruczynski, R. V. Gorbachev, T. Tudorovskiy, A. Zhukov, S. V. Morozov, M. I. Katsnelson, V. I. Fal'ko, A. K. Geim et al., Interaction-driven spectrum reconstruction in bilayer graphene, Science 333, 860 (2011).

[25] A. Varlet, D. Bischoff, P. Simonet, K. Watanabe, T. Taniguchi, T. Ihn, K. Ensslin, M. Mucha-Kruczynski, and V. I. Fal'ko, Anomalous Sequence of Quantum Hall Liquids Revealing a Tunable Lifshitz Transition in Bilayer Graphene, Phys. Rev. Lett. 113, 116602 (2014).

[26] H. Isobe, S. Y. Xu, and L. Fu, High-frequency rectification via chiral Bloch electrons, Sci. Adv. 6, eaay2497 (2020).

[27] T. Morimoto and N. Nagaosa, Nonreciprocal current from electron interactions in noncentrosymmetric crystals: Roles of time reversal symmetry and dissipation, Sci. Rep. 8, 2973 (2018).

[28] D. Xiao, W. Yao, and Q. Niu, Valley-Contrasting Physics in Graphene: Magnetic Moment and Topological Transport, Phys. Rev. Lett. 99, 236809 (2007).

[29] Graphene Nanoelectronics, edited by H. Raza, NanoScience and Technology (Springer-Verlag, Berlin, 2012), Chap. 8.

[30] M. Sui, G. Chen, L. Ma, W. Y. Shan, D. Tian, K. Watanabe, T. Taniguchi, X. Jin, W. Yao, D. Xiao, and Y. Zhang, Gate-tunable topological valley transport in bilayer graphene, Nat. Phys. 11, 1027 (2015). 
[31] Y. Shimazaki, M. Yamamoto, I. V. Borzenets, K. Watanabe, T. Taniguchi, and S. Tarucha, Generation and detection of pure valley current by electrically induced Berry curvature in bilayer graphene, Nat. Phys. 11, 1032 (2015).

[32] Y. D. Lensky, J. C. W. Song, P. Samutpraphoot, and L. S. Levitov, Topological Valley Currents in Gapped Dirac Materials, Phys. Rev. Lett. 114, 256601 (2015).

[33] Y. W. Tan, Y. Zhang, K. Bolotin, Y. Zhao, S. Adam, E. H. Hwang, S. Das Sarma, H. L. Stormer, and P. Kim, Measurement of Scattering Rate and Minimum Conductivity in Graphene, Phys. Rev. Lett. 99, 246803 (2007).
[34] S. Xiao, J. H. Chen, S. Adam, E. D. Williams, and M. S. Fuhrer, Charged impurity scattering in bilayer graphene, Phys. Rev. B 82, 041406(R) (2010).

[35] S. Das Sarma, E. H. Hwang, and E. Rossi, Theory of carrier transport in bilayer graphene, Phys. Rev. B 81, 161407(R) (2010).

[36] J. Li, L. Z. Tan, K. Zou, A. A. Stabile, D. J. Seiwell, K. Watanabe, T. Taniguchi, S. G. Louie, and J. Zhu, Effective mass in bilayer graphene at low carrier densities: The role of potential disorder and electron-electron interaction, Phys. Rev. B 94, 161406(R) (2016). 